\title{
Numerical study of the effects of contact angle and viscosity ratio on the dy- namics of snap-off through porous media
}

\author{
Authors \\ M. Starnoni*, D. Pokrajac \\ School of Engineering, University of Aberdeen \\ King's College, Aberdeen AB24 3UE Scotland, United Kingdom
}

*Corresponding author: m.starnoni@abdn.ac.uk, michele.starnoni@gmail.com

\section{Acknowledgements}

This work was jointly sponsored by EPSRC (EP/I010971/1) and NSFC China. The authors would like to acknowledge the support of the Maxwell compute cluster funded by the University of Aberdeen.

\section{Abstract}

Snap-off is a pore-scale mechanism occurring in porous media in which a bubble of nonwetting phase displacing a wetting phase, and vice-versa, can break-up into ganglia when passing through a constriction. This mechanism is very important in foam generation processes, enhanced oil recovery techniques and capillary trapping of $\mathrm{CO}_{2}$ during its geological storage. In the present study, the effects of contact angle and viscosity ratio on the dynamics of snap-off are examined by simulating drainage in a single pore-throat constriction of variable cross-section, and for different pore-throat geometries. To model the flow, we developed a CFD code based on the Finite Volume method. The Volume-of-fluid method is used to track the interfaces. Results show that the threshold contact angle for snap-off, i.e. snap-off occurs only for contact angles smaller than the threshold, increases from a value of $28^{\circ}$ for a circular cross-section to $30-34^{\circ}$ for a square cross-section and up to $40^{\circ}$ for a triangular one. For a throat of square cross-section, increasing the viscosity of the injected phase results in a drop in the threshold contact angle from a value of $30^{\circ}$ when the viscosity ratio $\bar{\mu}$ is equal to 1 to $26^{\circ}$ when $\bar{\mu}=20$ and down to $24^{\circ}$ when $\bar{\mu}=20$. 


\section{Keywords}

Numerical simulations, snap-off, contact angle, viscosity ratio, PLIC-VOF

\section{Introduction}

Multiphase fluid flow in porous media is encountered in many practical engineering problems, including oil, water and gas flow in petroleum reservoirs, and storage of Carbon Dioxide $\left(\mathrm{CO}_{2}\right)$ in deep underground aquifers. There are two types of displacement which can take place in two-phase systems: drainage, in which the wetting phase (water, in this work) is displaced by a non-wetting phase (typically a gas, for example $\mathrm{CO}_{2}$ ), and imbibition, in which the wetting phase is adsorbed into the porous medium and displaces the resident non-wetting phase. A dominant pore-scale mechanism occurring when a non-wetting phase is injected into a reservoir predominanly occupied by a wetting phase, and vice versa, is the break-up of the injected phase into several ganglia when passing through a constriction. This mechanism is called "snap-off" (Roof et al., 1970; Ransohoff et al., 1987; Gauglitz and Radke, 1990; Kovscek and Radke, 1996; Rossen, 2000). It is relevant to foam generation processes and capillary trapping of oil or $\mathrm{CO}_{2}$ bubbles during enhanced oil recovery or geological storage of $\mathrm{CO}_{2}$. Several studies have been conducted to analyze the snap-off mechanism in order to establish a criterion for its occurrence. The first quasi-static criterion was derived by Roof et al. (1970) for circular pores and for perfectly wetting conditions $\left(\theta=0^{\circ}\right)$. The criterion states that snap-off occurs when the capillary pressure at the bubble front in the unconstricted pore becomes less than the local capillary pressure at the throat, i.e. when the following inequality is satisfied:

$$
\frac{2}{R_{p}} \leq \frac{1}{R_{t}}+\frac{1}{R_{z t}}
$$

where $R_{p}$ and $R_{t}$ are the pore and throat radii and $R_{z t}$ is the longitudinal radius of curvature of the throat. In eq. (1), the left hand side is the mean curvature of the bubble front in the unconstricted section of the pore while the right hand side is the local curvature of the throat. Ransohoff et al. (1987) extended Roof's criterion to variable cross-sections as follows:

$$
\frac{\tilde{C}_{p}}{R_{p}} \leq \frac{1}{\tilde{R}_{t}}+\frac{1}{R_{z t}}
$$


where the coefficient $\tilde{C}_{p}$ depends on the shape of the cross-section $\left(\tilde{C}_{p}=2\right.$ for circular, 1.89 for square and 1.77 for triangular), $\tilde{R}_{t}$ is the radius of the largest inscribed circle at the throat and the r.h.s. is the critical curvature for snap-off at the throat, corresponding to the point of instability where the curvatures at two corners of the cross-section meet. For long enough throats, the above criterion reduces to

$$
R_{p} \geq \tilde{C}_{p} \tilde{R}_{t}
$$

This is a purely geometric criterion stating that snap-off occurs when the contraction ratio $C R$, defined as

$$
C R=\frac{R_{p}}{R_{t}}
$$

satisfies certain conditions. However, geometry-constrained snap-off, governed by Roof's criterion, is not the only mechanism for snap-off to occur (Rossen, 2003). Considering a capillary channel with sinusoidal shape, when the invading fluid becomes continuous within the channel, its break-up is determined by the imbalance between the pressure critical values at the necks and pores (Beresnev et al., 2009). To this extent, Beresnev and Deng (2010) developed a theory of fluid break-up in sinusoidally constricted capillary channels. Their formulation reduces to the condition for Rayleigh instability as a limiting case. Deng et al. (2014) used the latter model to study the snap-off of $\mathrm{CO}_{2}$ in capillary channels of such sinusoidal profile. They considered capillary channels with different wavelenghts but fixed contraction ratio. They then observed that snap off can occur even in capillary channels which do not meet Roof's criteria. Hence, they proposed a new criterion of the form

$$
\frac{\tilde{C}_{p}}{\tilde{R}_{p}}+\frac{1}{R_{z p}} \leq \frac{\tilde{C}_{t}}{\tilde{R}_{t}}+\frac{1}{R_{z t}}
$$

where $R_{z p}$ is the longitudinal radius of curvature of the pore. Raeini et al. (2014) investigated the existence of a threshold contraction ratio for snap-off for a single star-shaped pore-throat system of different aspect ratios $A R$

$$
A R=\frac{L_{t}}{R_{p}},
$$


where $L_{t}$ is the length of the throat. Finally Armstrong et al. (2016) used Density Functional Hydrodynamics (DFH) tools to study geometry-constrained snap-off in pore doublets and simple systems of pores, demonstrating consistency of simulation results with theoretical criteria. In particular, they compared the curvature at the leading interface measured from the numerical simulations against its theoretical value at the moment of snap-off from Roof's model. They observed slight discrepancy between these values due to the fact that theoretical models assume quasi-static conditions while inertial effects are present in numerical simulations. However, they also observed that this discrepancy tends to decrease with increasing grid resolution.

However, the last numerical studies assumed perfectly wetting behaviour between the two fluids, i.e. $\theta=0^{\circ}$. Little effort has been devoted to quantifying the influence of contact angle on the occurrence of snap-off. Yu and Wardlaw (1986) carried out experiments under quasi-static conditions on a single pore-throat system of square cross-section. They found that the critical contraction ratio for snap-off increases only slightly from 1.5 , when $\theta$ is equal to zero, to 1.75 , when $\theta=55^{\circ}$, however above $\sim 70^{\circ}$ snap-off never occurs. Porenetwork studies (Blunt et al., 1997; Mogensen and Stenby, 1998) indicated that snap-off is inhibited for $\theta>45^{\circ}$ when throats have a square cross-section.

In the present study, the effects of contact angle and viscosity on the dynamics of snap-off are examined. The dimensionless quantities describing the flow that will be used in the following are the Reynolds number $R e$, the capillary number $C a$ and the viscosity ratio $\bar{\mu}$, defined as:

$$
\begin{aligned}
R e & =\frac{\rho v D}{\mu}, \\
C a & =\frac{\mu v}{\sigma}, \\
\bar{\mu} & =\frac{\mu_{n w}}{\mu_{w}},
\end{aligned}
$$

where $v$ is the velocity, $\rho$ and $\mu$ are the fluid's density and viscosity, $D$ is the diameter of the pipe and $\sigma$ is the interfacial tension. The study is conducted by simulating drainage in a single pore-throat constriction of variable cross-section and for different pore-throat geometries. To model the flow, we developed the $\mathrm{C}++$ in-house code interpore $3 d$ which solves the governing equations by means of the Finite Volume (FV) method. The numer- 


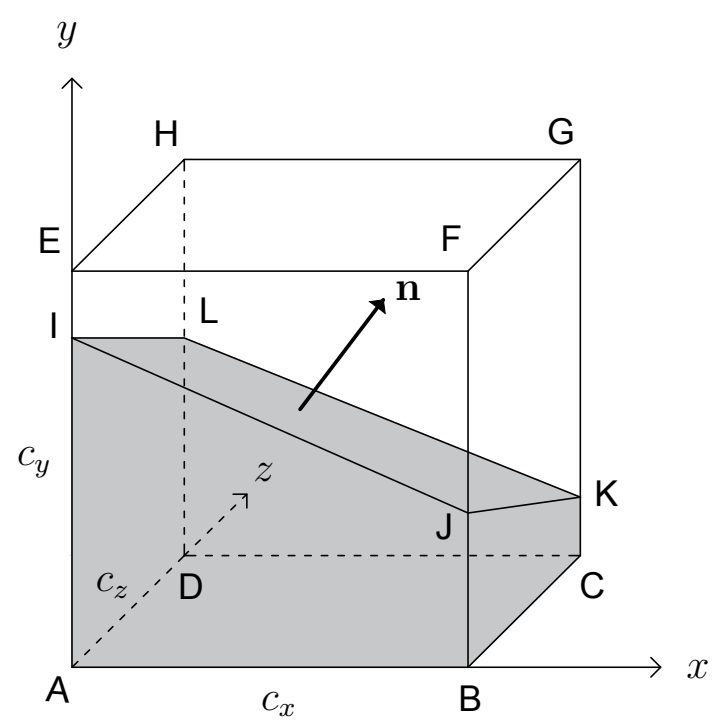

Figure 1. Standard configuration of the "truncated volume". All the three normal components are positive with fluid 1 laying below the interface

ical method is described in Sec. 2. Simulation results of benchmark problems for model validation are presented in Sec. 3. These include the classic static drop problem and simulations of imbibition in a circular pipe for different flow conditions. Finally, simulation results of snap-off are presented in Sec. 4.

\section{$2 \quad$ Numerical method}

The method consists of three main components: an interface-tracking algorithm, a model for the surface tension forces and a solver for the incompressible Navier-Stokes (NS) equations.

\subsection{The interface-tracking algorithm}

The interfaces are tracked using the Volume-of-Fluid (VOF) method by Hirt and Nichols (1981). The VOF method is a volume-tracking method for the representation of the interfaces in interfacial flow problems, in which the fluids are marked and tracked by means of a volume fraction, also called indicator function. In a VOF-FV framework, the indicator function $\alpha$ is defined for each cell as the ratio between the volume of fluid 1 contained within the cell, $V^{\alpha}$, and the volume of the cell $V$ as

$$
\alpha=\frac{V^{\alpha}}{V}
$$


This means that the indicator function is bounded between 0 and 1 as follows

$$
\alpha= \begin{cases}1, & \text { if the cell is filled with fluid } 1 \\ 0, & \text { if the cell is filled with fluid } 2 \\ 0<\alpha<1, & \text { if there is an interface within the cell. }\end{cases}
$$

Starting from a known initial distribution, the volume fraction is updated in time by solving the following advection equation

$$
\frac{\partial \alpha}{\partial t}+\nabla \cdot(\alpha \mathbf{u})=\alpha \nabla \cdot \mathbf{u}
$$

where $t$ is time and $\mathbf{u}$ is the velocity vector. Advection is done through operators-splitting, i.e the interface is advanced in time with three independent sweeps along each of the three directions, where the order of the sweeps is alternated at each time step to ensure better stability. We employed the Piecewise Linear Interface Calculation (PLIC) method by Youngs (1982) to solve eq. (10) numerically. In the PLIC method, the interface is mathematically described as a planar surface defined by equation

$$
\mathbf{n} \cdot \mathbf{x}-\beta=0
$$

where $\beta$ is a constant and $\mathbf{n}$ is the interface unit normal vector pointing outwards from fluid 1:

$$
\mathbf{n}=-\frac{\nabla \alpha}{|\nabla \alpha|}
$$

The minus sign in eq. (12) comes from the choice of the normal pointing direction. Discretization of the gradient operator in eq. (12) is done using a 27 points-stencil Finite Difference scheme, while determination of the constant $\beta$ is done by matching the interface truncated volume $V(\mathbf{n}, \beta)$ (the volume ABCDIJKL in Fig. 1) to the actual fluid volume $V^{\alpha}$ contained in the cell using Brent's method (Press et al., 1996). A useful formula for the truncated volume is given by Gueyffier et al. (1999). Further detail on the PLIC algorithm can be found in Kothe et al. (1996) and Rider and Kothe (1998). 

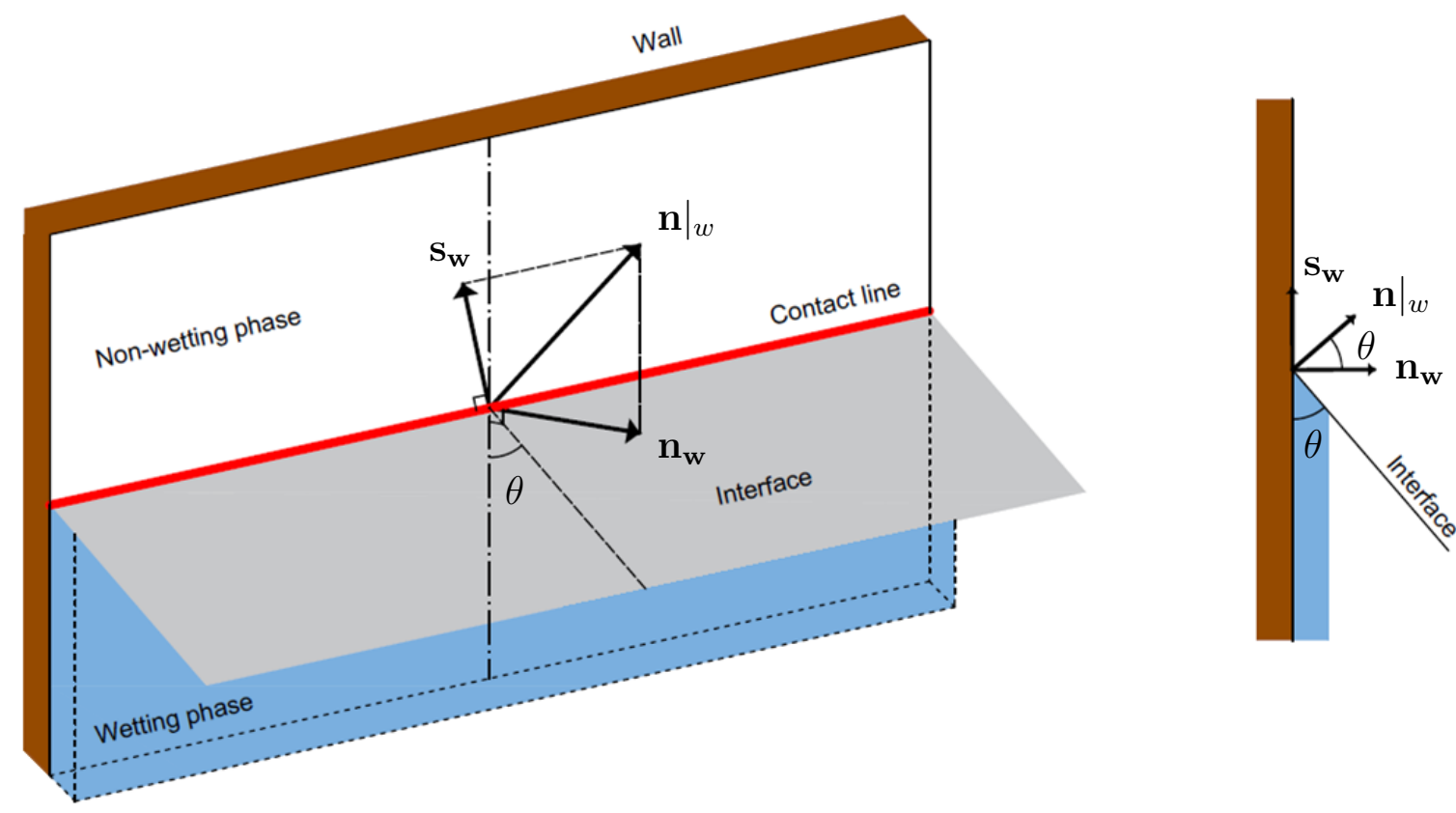

Figure 2. Unit vectors at the contact line between a solid wall and an interface: $\left.\mathbf{n}\right|_{w}$ is the normal to the interface at the contact line, $\mathbf{n}_{\mathbf{w}}$ is the unit normal to the wall pointing outwards from the wall and $\mathbf{s}_{\mathbf{w}}$ is a unit vector lying in the wall and normal to the contact line. $\theta$ is the contact angle between the interface and the solid wall, measured towards the wetting phase.

\subsection{The incompressible Navier-Stokes equations with surface tension forces}

The Navier-Stokes equations with surface tension forces are written as

$$
\frac{\partial}{\partial t}(\rho \mathbf{u})+\nabla \cdot(\rho \mathbf{u u})+\nabla p-\nabla \cdot \tau-\rho \mathbf{g}-\mathbf{f}_{s}=0
$$

where $p$ and $\mathbf{u}=(u, v, w)$ are the pressure and velocity respectively, $\tau=\mu\left[\nabla \mathbf{u}+(\nabla \mathbf{u})^{T}\right]$ is the viscous stress tensor, $\mathbf{g}$ is the gravitational acceleration, $\mathbf{f}_{s}$ is the surface tension force and $\rho$ and $\mu$ are the fluid density and viscosity, which in the VOF method are computed as

$$
\begin{aligned}
& \rho=\alpha \rho_{1}+(1-\alpha) \rho_{2}, \\
& \mu=\alpha \mu_{1}+(1-\alpha) \mu_{2} .
\end{aligned}
$$

The use of the VOF method for the representation of the interface between two immiscible fluids offers the advantage that the numerical techniques for the solution of the NS 
equations are the same for two-phase systems as in single-phase flow. The two-phase flow problem can thus be seen as the flow of a single fluid with spatially and temporally variable physical properties. However, the presence of localized interfaces generates additional forces of chemical nature due to the change of molecular forces in the transition region between the two fluids. These forces are embodied in the NS equations as body forces concentrated at the interface, i.e. the term $\mathbf{f}_{\mathbf{s}}$ in eq. (13), and their discretization must be treated carefully. Following the Continuous Surface Force (CSF) model by Brackbill et al. (1992), the surface tension force $\mathbf{f}_{s}$ is computed as a body force using the Dirac delta function $\delta_{s}$ concentrated at the interface as follows:

$$
\mathbf{f}_{s}=\sigma k \mathbf{n} \delta_{s}
$$

where $\sigma$ is the interfacial tension, $k$ is the interface curvature defined as (Aris, 1962)

$$
k=-\nabla \cdot \mathbf{n}
$$

and $\mathbf{n}$ is the unit normal to the interface computed using eq. (12). For those interface cells which are in direct contact with a solid wall boundary (see Fig. 2), the normal takes the form (Brackbill et al., 1992)

$$
\left.\mathbf{n}\right|_{w}=\mathbf{n}_{\mathbf{w}} \cos \theta+\mathbf{s}_{\mathbf{w}} \sin \theta
$$

where $\theta$ is the contact angle measured between the wall and the wetting phase, $\mathbf{n}_{\mathbf{w}}$ is the unit normal to the wall pointing outwards from the wall and $\mathbf{s}_{\mathbf{w}}$ is a vector lying in the wall and normal to the contact line given by (Qaseminejad Raeini, 2013)

$$
\mathbf{s}_{\mathbf{w}}=\frac{\mathbf{n}-\left(\mathbf{n} \cdot \mathbf{n}_{\mathbf{w}}\right) \mathbf{n}_{\mathbf{w}}}{\left|\mathbf{n}-\left(\mathbf{n} \cdot \mathbf{n}_{\mathbf{w}}\right) \mathbf{n}_{\mathbf{w}}\right|}
$$

Eq. (17) replaces eq. (12) in the interface tracking algorithm for those interface cells which are in direct contact with a solid wall boundary, i.e. cells with indicator function $\alpha$ between 0 and 1, having at least one solid wall cell among the direct adjacent cells.

The NS equations are solved using the PISO algorithm (Issa, 1986). We employed the Filtered Surface Force (FSF) formulation by Raeini et al. (2012) for filtering the 

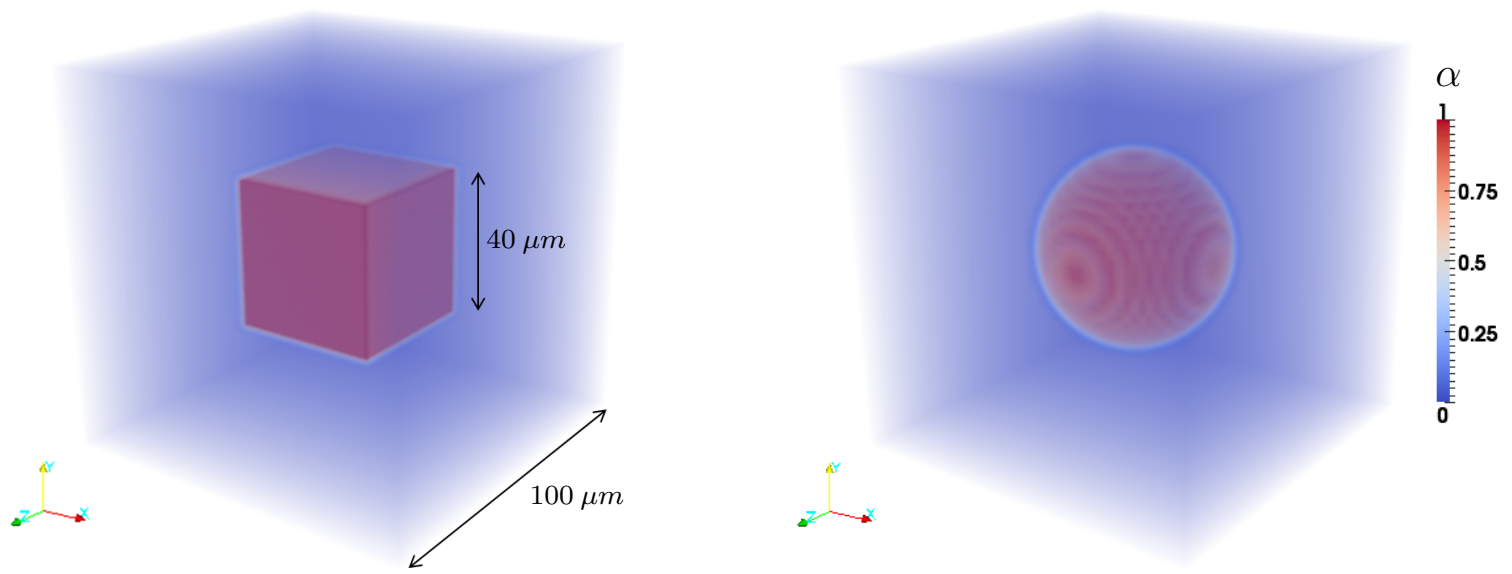

Figure 3. Static drop test: initial (a) and final (b) volume fraction distribution for the 3D drop with $L / \Delta x=16$

surface tension forces in the interface transition region. All the resulting linear systems of equations are solved using the PBCG method with incomplete LU factorization as preconditioner (Saad, 2003). Finally, there is a stability condition to respect when solving the NS equations with surface tension forces, i.e. the numerical method is stable when the time step resolves the capillary wave propagation (Brackbill et al., 1992)

$$
\Delta t<\sqrt{\frac{\langle\rho\rangle \Delta x^{3}}{2 \pi \sigma}}
$$

where $\langle\rho\rangle$ is the average density of the two fluids and $\Delta x$ is the characteristic grid size. In order to facilitate the presentation of the numerical setup for the various tests where surface tension forces are involved, a modified time step constraint is introduced:

$$
\Delta t=C_{t} \Delta t_{b k z}
$$

where $C_{t}$ is a constant and $\Delta t_{b k z}$ is the r.h.s. of eq. (19).

\section{Validation}

\subsection{Surface tension test: static drop}

This is the typical test which is commonly used for validating surface tension implementations. A problem often arising in numerical simulations when accounting for surface 


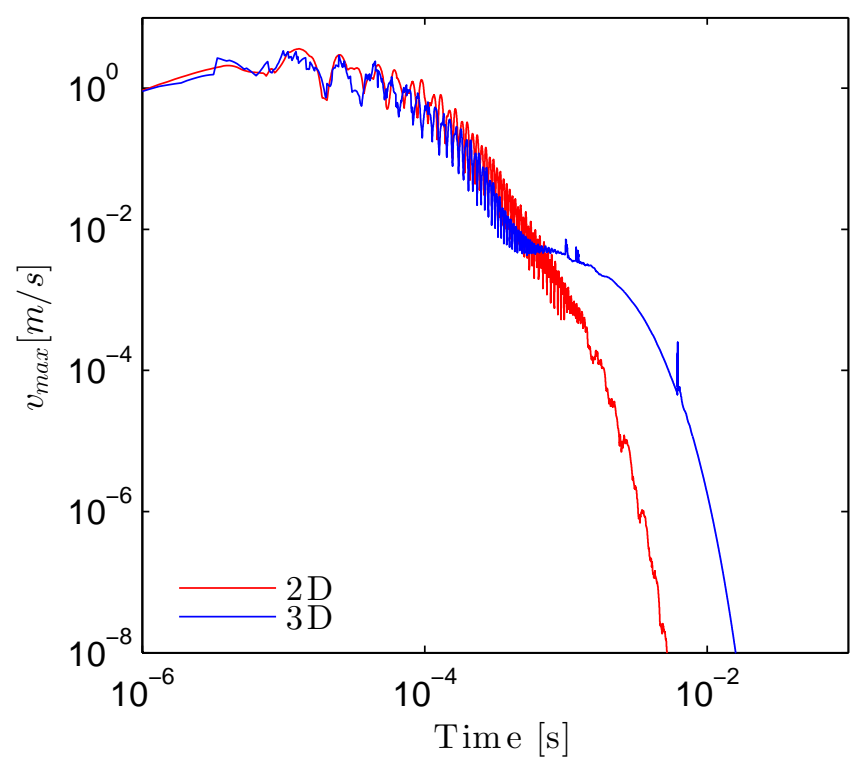

Figure 4. Static drop test: computed max velocity in the computational domain as a function of time $(L / \Delta x=16)$

tension effects is the occurrence of the so-called "spurious currents", a non-vanishing velocity field which appears in the interface transition region even in absence of external forces (Lafaurie et al., 1994; Brackbill and Kothe, 1996; Williams et al., 1998; Scardovelli and Zaleski, 1999; Renardy and Renardy, 2002; Sussman and Ohta, 2006; Raeini et al., 2012). Here, both a 2D and a 3D bubble of gas immersed in another fluid approaching its equilibrium state in absence of gravity are studied. The gas has viscosity $\mu_{g}=10^{-5} \mathrm{~Pa} \cdot \mathrm{s}$ and density $\rho_{g}=1 \mathrm{~kg} / \mathrm{m}^{3}$ while the other fluid is water with viscosity $\mu_{w}=10^{-3} \mathrm{~Pa} \cdot \mathrm{s}$ and density $\rho_{w}=1000 \mathrm{~kg} / \mathrm{m}^{3}$. The interfacial tension coefficient $\sigma=0.07 \mathrm{~N} / \mathrm{m}$. Initially the bubble has square (2D) and cubic (3D) shape of size $\mathrm{L}=40 \mu \mathrm{m}$ and is placed at the centre of a computational domain of size 2.5L to allow for the bubble deformation. This setup is chosen for comparison with the work of Raeini et al. (2012). Velocity is set to zero everywhere at the boundary. Simulations with $C_{t}=0.5$ are carried out for different resolutions $L / \Delta x=3,4,8,16$ and 32 .

The aim of this test is to prove that the model is able to eliminate the so-called spurious currents, as in Raeini et al. (2012), and to show the convergence of the bubble to its exact curvature:

$$
k_{\text {exact }}=\left\{\begin{array}{llll}
1 / r, & \text { with } & r=\sqrt{\frac{A_{\alpha}^{f}}{\pi}}, & 2 \mathrm{D} \\
2 / r, & \text { with } & r=\sqrt[3]{\frac{3 V_{\alpha}^{f}}{4 \pi}}, & 3 \mathrm{D}
\end{array}\right.
$$




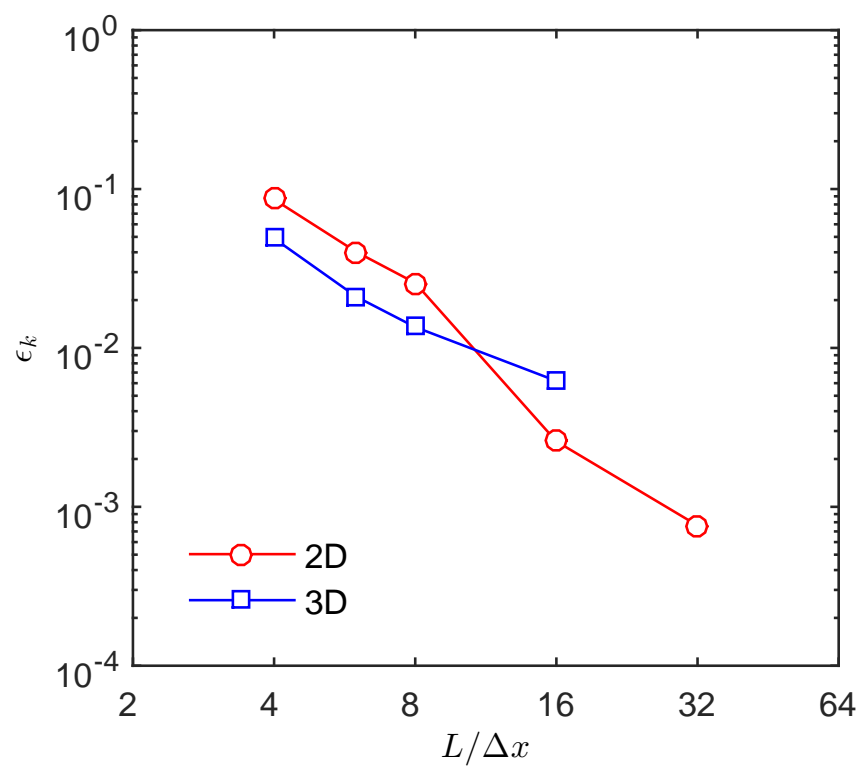

Figure 5. Static drop test: error in the drop curvature as a function of grid resolution where $A_{\alpha}^{f}$ and $V_{\alpha}^{f}$ are the bubble area and volume respectively in the final equilibrium configuration. The initial and final equilibrium configurations can be visualized in Fig. 3. As far as eliminating the spurious currents is concerned, Fig. 4 clearly shows how the velocity eventually tends to zero everywhere in the domain. This was the main goal of the test. The error in the curvature is measured as

$$
\epsilon_{k}=\frac{\left|k_{\text {num }}-k_{\text {exact }}\right|}{k_{\text {exact }}}
$$

where the numerical curvature $k_{n u m}$ is computed using a weighted average as:

$$
k_{n u m}=\frac{\sum k_{i}\left|\nabla \alpha^{f}\right|_{i} V_{i}}{\sum\left|\nabla \alpha^{f}\right|_{i} V_{i}}
$$

The error in the curvature plotted as a function of grid resolution is shown in Fig. 5, exhibiting convergence towards zero with increasing grid resolution.

\subsection{Imbibition in a circular capillary pipe}

The movement of a ganglion of water displacing another fluid $(\bar{\mu}=1,0.01)$ in a circular pipe of radius $R$ and length $L=8 R$ is studied. The fluids physical properties are the same as in simulations presented in Sec. 3.1, with interfacial tension coefficient $\sigma=0.05$ $N / m$, the static contact angle $\theta=45^{\circ}$ and the grid resolution $R / \Delta x=5$. This test aims at investigating the stability of the numerical method for different flow conditions and 
assessing its capability to handle high density and viscosity ratios in dynamic conditions.

Boundary conditions consist of pressure at the outlet and a Poiseuille velocity distribution at the inlet $v_{i n}$. The Reynolds and capillary number are controlled by varying the mean inlet velocity $\bar{v}_{\text {in }}=0.1,0.01$ and $0.001 \mathrm{~m} / \mathrm{s}$ and the pipe diameter $D=25,250$, $2500 \mu \mathrm{m}$, yielding a set of 3x3 possible flow conditions, with $C a$ and $R e$ ranging between $2.0 e^{-3}$ and $2.0 e^{-5}$ and between 0.025 and 250 respectively.

Initially, the interface is placed at $L / 4$ and a static simulation is carried out in order to start the dynamic simulations from equilibrium conditions. $C_{d t}$ is set equal to 1 for the case with $\bar{\mu}=1$ and 0.5 for the case with $\bar{\mu}=0.01$. All the simulations are run until breakthrough or stopped after 200000 time steps.

The stability and accuracy of the simulations are evaluated using several parameters: the maximum difference in curvature $\epsilon_{k}$, the maximum difference in average interface velocity $\epsilon_{v}$, the relative standard deviation of the average interface velocity $R S D_{v}$ and the error in mass conservation $\epsilon_{m c}$. The maximum difference in curvature $\epsilon_{k}$ is the largest discrepancy between the curvature computed after each time step using eq. (23) and the exact one:

$$
\epsilon_{k}=\max \left(\frac{\left|k_{\text {min }}-k_{\text {exact }}\right|}{k_{\text {exact }}}, \frac{\left|k_{\text {max }}-k_{\text {exact }}\right|}{k_{\text {exact }}}\right) \text {, }
$$

where $k_{\min }$ and $k_{\max }$ are the minimum and maximum curvature recorded during the simulation, and $k_{\text {exact }}$ is:

$$
k_{\text {exact }}=\frac{2 \sigma \cos \theta}{R}
$$

The maximum difference in the average interface velocity $\epsilon_{v}$ is computed in the same way as

$$
\epsilon_{v}=\max \left(\frac{\left|\bar{v}_{s, \min }-\bar{v}_{i n}\right|}{\bar{v}_{i n}}, \frac{\left|\bar{v}_{s, \max }-\bar{v}_{i n}\right|}{\bar{v}_{i n}}\right),
$$

where $\bar{v}_{s}$ is the mean interface velocity, i.e. the average velocity calculated for the interface cells, after each time step. The relative standard deviation of the average interface velocity $R S D_{v}$ is a measure of the variability of the average interface velocity during the simulations. It is defined as the ratio between its standard deviation and mean value during the whole computation. Finally, the mass conservation error is the relative difference between the total injected volume at the end of the simulation $V_{i n}=\left(t^{f}-t^{0}\right) \int_{\partial A} v_{i n} d A$ 
Table 1. Measures of error for different capillary and Reynolds numbers, and for different pair of fluids.

\begin{tabular}{llclllr}
\hline$C a$ & $R e$ & $\bar{\mu}$ & $\epsilon_{k}$ & $\epsilon_{v}$ & $R S D_{v}$ & $\epsilon_{m c}$ \\
\hline \multirow{2}{*}{$2 \mathrm{e}-3$} & 2.5 & 1 & 0.061 & 0.23 & 0.041 & $5.95 \mathrm{e}-4$ \\
& & 0.01 & 0.046 & 0.37 & 0.044 & $1.56 \mathrm{e}-3$ \\
$2 \mathrm{e}-3$ & 25 & 1 & 0.077 & 0.11 & 0.033 & $1.32 \mathrm{e}-3$ \\
& & 0.01 & 0.055 & 0.37 & 0.053 & $1.52 \mathrm{e}-3$ \\
$2 \mathrm{e}-3$ & 250 & 1 & 0.063 & 0.11 & 0.033 & $3.60 \mathrm{e}-3$ \\
& & 0.01 & 0.076 & 0.39 & 0.030 & $6.21 \mathrm{e}-3$ \\
$2 \mathrm{e}-4$ & 0.25 & 1 & 0.045 & 0.47 & 0.087 & $3.50 \mathrm{e}-5$ \\
& & 0.01 & 0.039 & 0.57 & 0.083 & $2.86 \mathrm{e}-4$ \\
$2 \mathrm{e}-4$ & 2.5 & 1 & 0.041 & 0.63 & 0.089 & $6.05 \mathrm{e}-5$ \\
& & 0.01 & 0.037 & 0.55 & 0.098 & $2.03 \mathrm{e}-3$ \\
$2 \mathrm{e}-4$ & 25 & 1 & 0.047 & 0.68 & 0.102 & $4.04 \mathrm{e}-5$ \\
& & 0.01 & 0.046 & 0.44 & 0.061 & $8.64 \mathrm{e}-3$ \\
$2 \mathrm{e}-5$ & 0.025 & 1 & 0.056 & 1.60 & 0.156 & $1.05 \mathrm{e}-3$ \\
& & 0.01 & 0.043 & 3.25 & 0.159 & $1.30 \mathrm{e}-3$ \\
$2 \mathrm{e}-5$ & 0.25 & 1 & 0.060 & 1.79 & 0.164 & $1.11 \mathrm{e}-3$ \\
& & 0.01 & 0.054 & 2.26 & 0.172 & $2.00 \mathrm{e}-3$ \\
$2 \mathrm{e}-5$ & 2.5 & 1 & 0.040 & 1.25 & 0.303 & $1.61 \mathrm{e}-3$ \\
& & 0.01 & 0.039 & 1.32 & 0.154 & $9.42 \mathrm{e}-3$ \\
\hline
\end{tabular}

and the simulated change in volume of the injected phase $\Delta V=V_{\alpha}^{f}-V_{\alpha}^{0}$ :

$$
\epsilon_{m c}=\frac{\left|\Delta V-V_{i n}\right|}{V_{i n}}
$$

where $V_{\alpha}^{f}$ and $V_{\alpha}^{0}$ are calculated from the initial and final volume fraction distributions in the computational domain.

As the computed parameters listed in Tab. 1 clearly indicate, all the simulations can be considered stable and accurate. The error in mass conservation is always below $1 \%$, confirming the robustness of the code in handling high density and viscosity ratios in dynamic conditions and at low capillary numbers. The largest maximum difference in curvature for all computations is $7.7 \%$. This means that the interface is pushed to advance uniformly through the pipe while maintaining its equilibrium configuration (see Fig. 6). The maximum difference in average interface velocity is generally higher than the other indicators, due to the localized surface tension forces active in the interface transition region and their interplay with the viscous forces active at the wall. A small numerical error in forces is likely to generate some local perturbations of the interface front, resulting in changes of the pressure jump across the interface and thus changes of the velocity field. The influence of the different flow conditions on the velocity field is also 

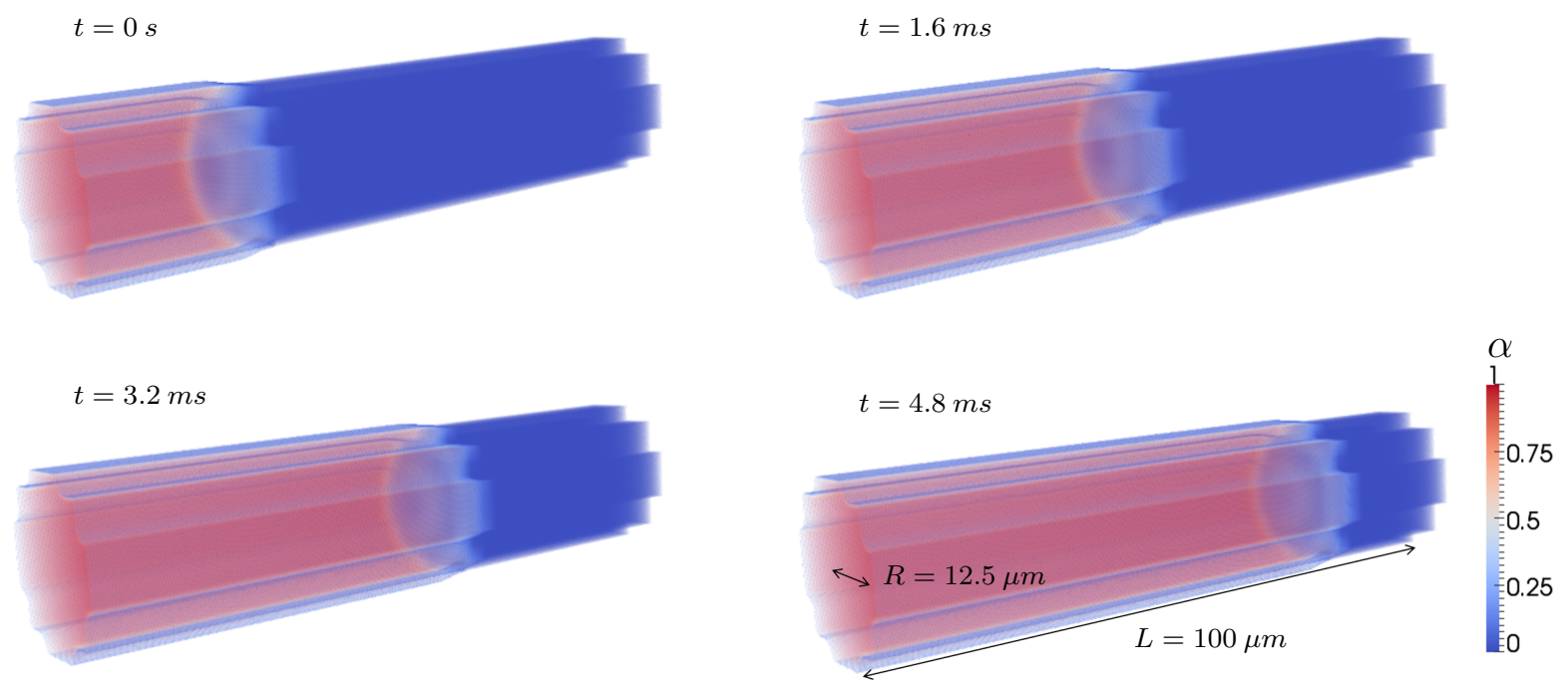

Figure 6. Time evolution of the volume fraction $\left(\bar{\mu}=0.01, C a=2 e^{-4}, R e=0.25\right)$.

more visible than their influence on the other two indicators. This is reasonable, as the curvature is primarily determined by the static contact angle $\theta$, which is not influenced by the flow conditions (hysteresis and dynamic effects are not considered in the model), while the mass conservation is mainly a feature of the advection scheme. First thing to note is the dramatic increase $\epsilon_{v}$ and $R S D_{v}$ at low capillary numbers. Again this can be explained by the local perturbations of the interface front due to surface tension forces. At higher capillary numbers capillary forces are less dominant with respect to viscous forces and the displacement is stabilized by viscous effects. On the contrary, at low capillary numbers capillary forces are dominant, resulting in a more unstable interface advancing front. This difference is exacerbated at high viscosity ratios. This is made clearer by visualizing snapshots of the velocity field in a plane parallel to the flow direction passing through the centre of the pipe (see Fig. 7). Far from the interface, the velocity assumes the Poiseuille distribution for single-phase flow. In the transition region, the capillary forces are responsible for aligning the wetting fluid velocities with the interface, hence moving the wetting fluid particles towards the walls. Evidence of these flow patterns was also shown by Molecular Dynamics (MD) simulations (Dimitrov et al., 2007). This results in a decrease in the velocity at the centre of the pipe compared to the Poiseuille distribution (see also Levine et al. (1980) for a study on the deviation from Poiseuille flow at the front meniscus). This in turn yields, for continuity, a higher velocity near the walls. For the system with high viscosity ratio this increase of the velocity at the contact line is more pronounced, giving rise to the formation of some vortices at the centre of 
(a)

$t=0.8 \mathrm{~ms}$

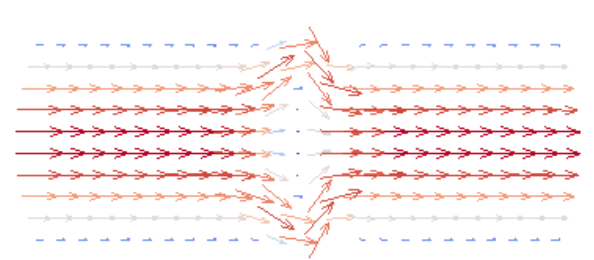

$t=1.4 \mathrm{~ms}$

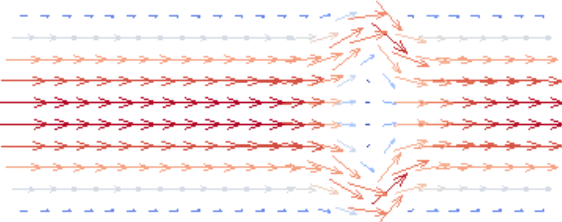

(b)
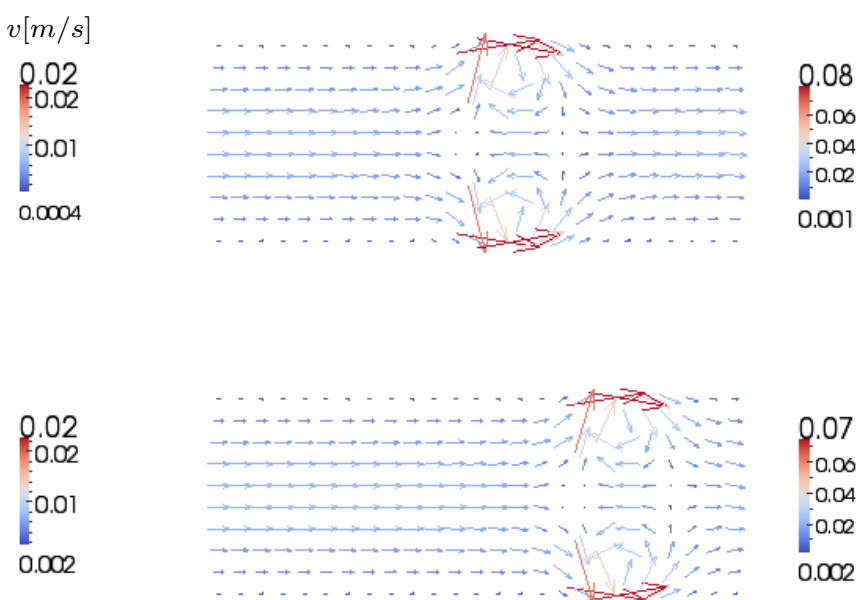

Figure 7. Snapshots of the velocity field near the interface along the central plane at different time steps for different values of the viscosity ratio: $\bar{\mu}=1$ (left column) and $\bar{\mu}=0.01$ (right column). $\left(C a=2 e^{-4}, R e=0.25\right)$.

the pipe (see Fig. 7b). This return flow is due to continuity because of the very high velocities next to the walls. However, it is likely that these very high velocities and thus the vortices are provoked by numerical instabilities in the transition region caused by the high viscosity ratios.

\subsection{Roof's criterion for snap-off}

Here the code is validated against Roof's extended criterion for snap-off, eq. (5). The constriction has the sinusoidal shape given by (Ransohoff et al., 1987)

$$
\frac{R(z)}{R_{p}}=1-\left(\frac{C R-1}{2 \cdot C R}\right)\left[1+\cos \left(2 \pi \frac{z}{\ell}\right]\right.
$$

where $R(z)$ is the constriction radius, $\mathrm{z}$ is the axial position measured from the neck of the constriction and $\ell$ is the constriction wavelength. For this shape function, Ransohoff et al. (1987) provided also an expression for the longitudinal radius of the curvature $R_{z t}$, which for circular pores is

$$
R_{z t}=\frac{\ell^{2}}{R_{p}}\left(\frac{C R}{2 \pi^{2}(C R-1)}\right)
$$

The same setup of Deng et al. (2014) is used. The contraction ratio $C R$ is fixed and equal to 2 ( $R_{p}$ and $R_{t}$ measure 100 and $50 \mu \mathrm{m}$ respectively), and two wavelengths are considered, $\ell_{1}=200$ and $\ell_{2}=600 \mu \mathrm{m}$. Circular pores and perfectly wetting conditions 
(a) $\ell_{1}$
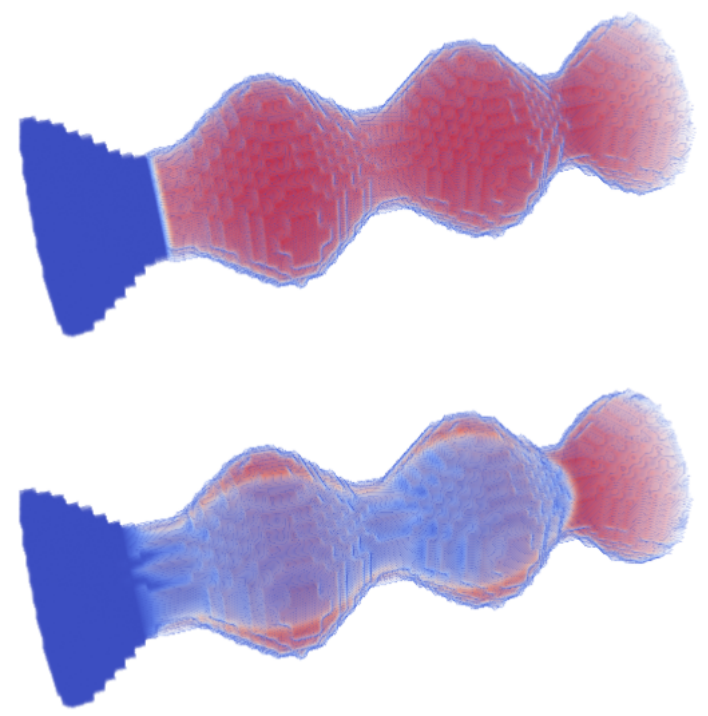

(b) $\ell_{2}$

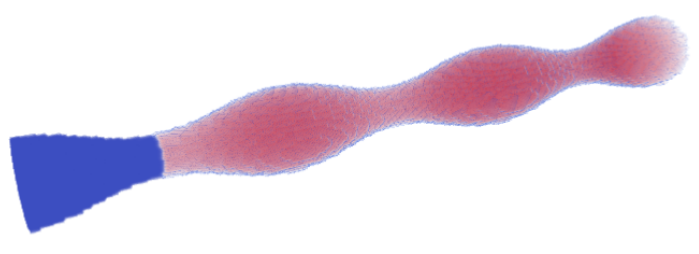

Figure 8. Initial (top) and final (bottom) volume fraction distribution for the sinusoidal constrictions with different wavelengths.

$(\theta=0)$ are considered. According to Roof's criteria, snap-off is expected to occur for the configuration with wavelength equal to $\ell_{2}$, while it is not expected to occur for the configuration with shorter wavelength $\ell_{1}$. The resident fluid is water, the interfacial tension coefficient is equal to $0.05 \mathrm{~N} / \mathrm{m}$ and the viscosity ratio is equal to 1 . Boundary conditions are the same as in simulations presented in Sec. 3.2, with inlet velocity equal to $5 \mathrm{~mm} / \mathrm{s}$, resulting in a capillary number equal to $1.0 e^{-4}$. Initially the interface is placed at the neck of the constriction and the inlet is entirely filled with the injected fluid. The grid resolution is $R_{t} / \Delta x=5$ and $C_{d t}=1$.

The initial and final fluid configurations for the two wavelengths considered can be visualized in Fig. 8. Results are in agreement with the extended Roof's criterion for snap-off, with snap-off happening only for the configuration with longer wavelength $\ell_{2}$.

\section{Numerical simulations}

\subsection{Numerical setup}

We study a single pore-throat constriction with four different configurations. The geometry of the problem is controlled solely by the contraction ratio $C R$ and the aspect ratio $A R$, defined in eqs. (4) and (6) respectively. Medium long $(A R=1.5)$ and long $(A R=5)$ throats, as well as medium narrow $(C R=2)$ and narrow $(C R=3)$ constrictions are 

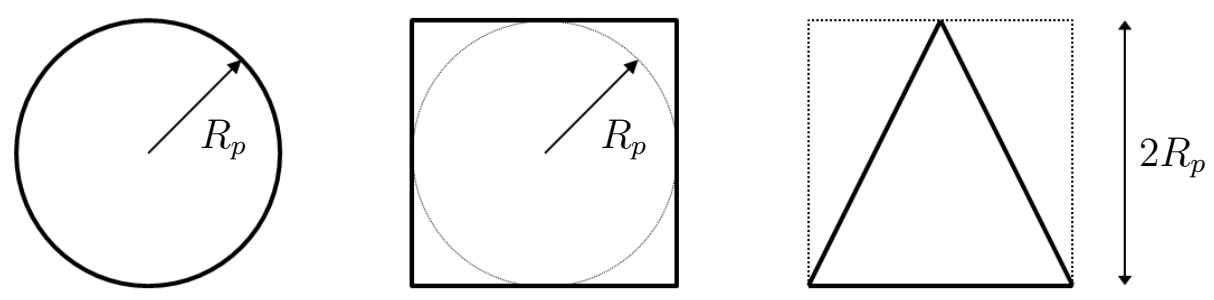

Figure 9. Cross-sections used in this study.

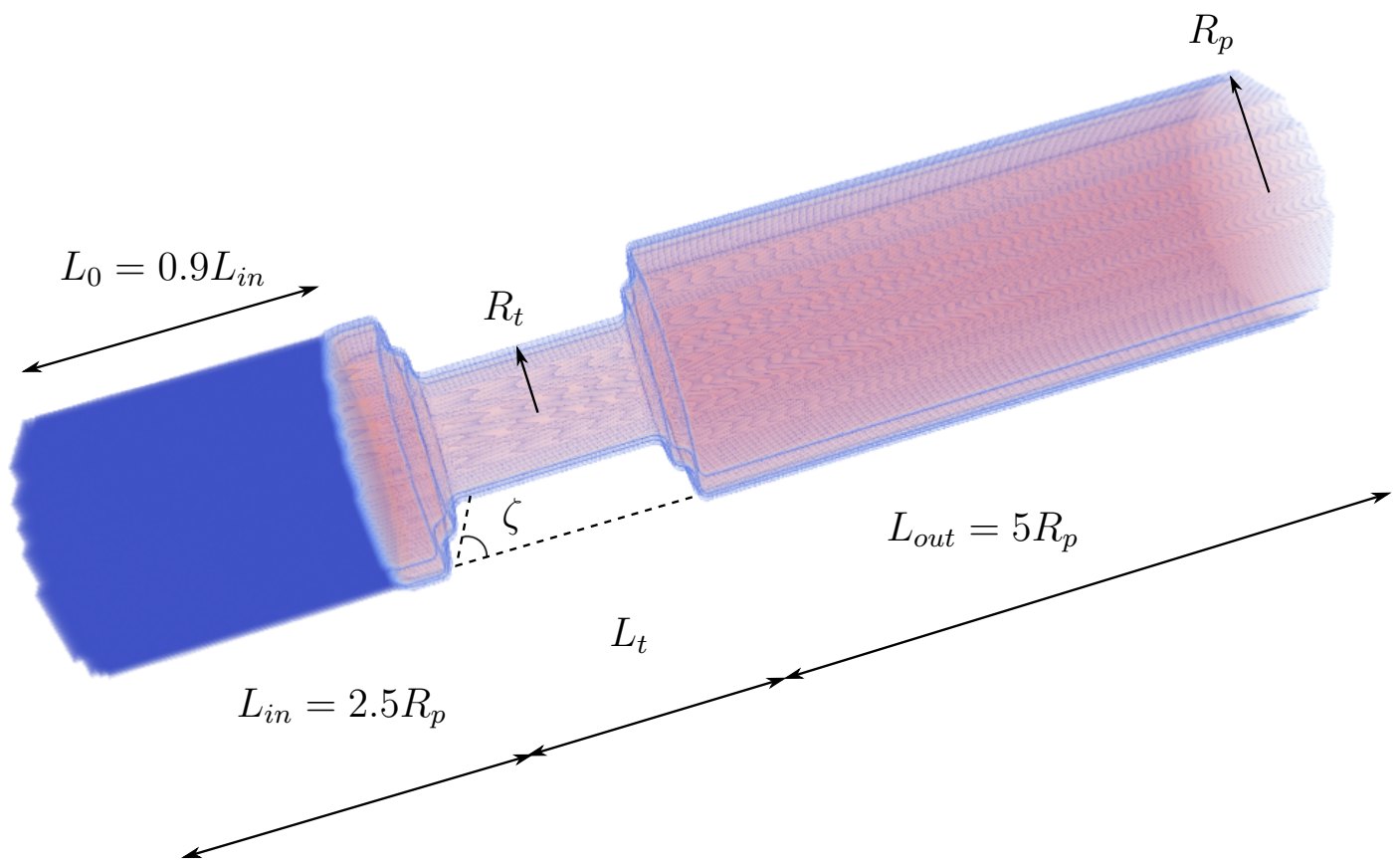

Figure 10. Initial conditions for the VOF indicator function (the non-wetting fluid is displayed in blue at the inlet) and the relevant geometrical parameters.

considered. We use three cross-sections (see Fig. 9): circular with unconstricted pore radius $R_{p}$ of $215 \mu \mathrm{m}$, square with largest inscribed circle in the unconstricted pore of radius equal to $R_{p}$ and isosceles triangle having base and height equal to $2 R_{p}$. This particular value of $R_{p}$ has been chosen to be consistent with the experimental apparatus of $\mathrm{Yu}$ and Wardlaw (1986), and is kept constant. The inlet and outlet pore lengths $L_{\text {in }}$ and $L_{\text {out }}$ are set equal to $2.5 R_{p}$ and $5 R_{p}$ respectively. Initially, the non-wetting fluid is positioned just at the throat entrance, at a distance of $0.9 L_{\text {in }}$ from the inlet. Boundary conditions are the same as in simulations presented in Sec. 3.2, with inlet velocity equal to $5 \mathrm{~mm} / \mathrm{s}$. Water is used as displaced wetting fluid, and the interfacial tension coefficient is always equal to $0.05 \mathrm{~N} / \mathrm{m}$, resulting in a capillary numer equal to $1.0 e^{-4}$. Finally, values of $C_{t}=1$ are used in all the simulations. The initial conditions for the VOF indicator function, as well as the various geometrical parameters involved, are displayed in Fig. 10.

Two series of simulations are performed. During the first series, the viscosity ratio 
Table 2. Summary of all cases used in the simulations. For each case, $\theta$ is varied from $0^{\circ}$ until snap-off is inhibited. Legend: $\mathrm{S}=$ square, $\mathrm{C}=$ circular, $\mathrm{T}=$ triangular.

\begin{tabular}{llllr}
\hline Case & Cross-section & $A R$ & $C R$ & $\bar{\mu}$ \\
\hline 1.1 & S, C, T & 1.5 & 2 & 1 \\
1.2 & S, C, T & 1.5 & 3 & 1 \\
1.3 & S, C, T & 5 & 2 & 1 \\
1.4 & S, C, T & 5 & 3 & 1 \\
2 & S & 1.5 & 2 & $0.02,1,10,20$ \\
\hline
\end{tabular}

is kept constant $(\bar{\mu}=1)$ and the effects of different cross-section and contact angle are investigated. All range of values of $\theta$ from $0^{\circ}$ until snap-off is inhibited are considered. The threshold contact angle for snap-off, $\theta_{\text {thresh }}$, above which snap-off is inhibited, is computed using a systematic trial-error procedure by changing the static contact angle and repeating the simulations. Simulations are stopped when snap-off occurs or at breakthrough. In order to automatically detect snap-off, a function is implemented which measures how many separate ganglia of non-wetting fluid are present within the system. When the number of independent ganglia is greater than one, the simulation is terminated and the static contact angle increased.

The second series is performed for the single case of square cross-section with $A R=1.5$ and $A R=2$ and $\theta_{\text {thresh }}$ is calculated for different viscosity ratios. The viscosity ratio is varied considering different injected fluid: oil having the same density of water and supercritical $\mathrm{CO}_{2}$ with density equal to $500 \mathrm{~kg} / \mathrm{m}^{3}$ and viscosity of $2 e^{-5} \mathrm{~Pa} \cdot \mathrm{s}$ (reference values taken from Huppert and Neufeld (2014)). A summary of the input data for the simulations is provided in Tab. 2.

\subsection{Mesh sensitivity}

Preliminary simulations were run for the standard case with square cross-section, $A R=$ 1.5, $C R=2$ and $\bar{\mu}=1$ in order to assess the role of some minor parameters such as the shape of the pore-throat junction, i.e. the angle $\zeta$ in Fig. 10, and the grid resolution $R_{t} / \Delta x$. While the shape of the pore-throat junction has no effect on the outcome of the simulations, the grid resolution strongly affects the dynamics of the flow. Fig. 11 shows the predicted pressure drop between the inlet and the outlet and the average capillary pressure as a function of time, for different values of grid resolution and for two values of $\theta$ equal to 24 and $32^{\circ}$ respectively. These values of $\theta$ correspond to cases whether snap-off 

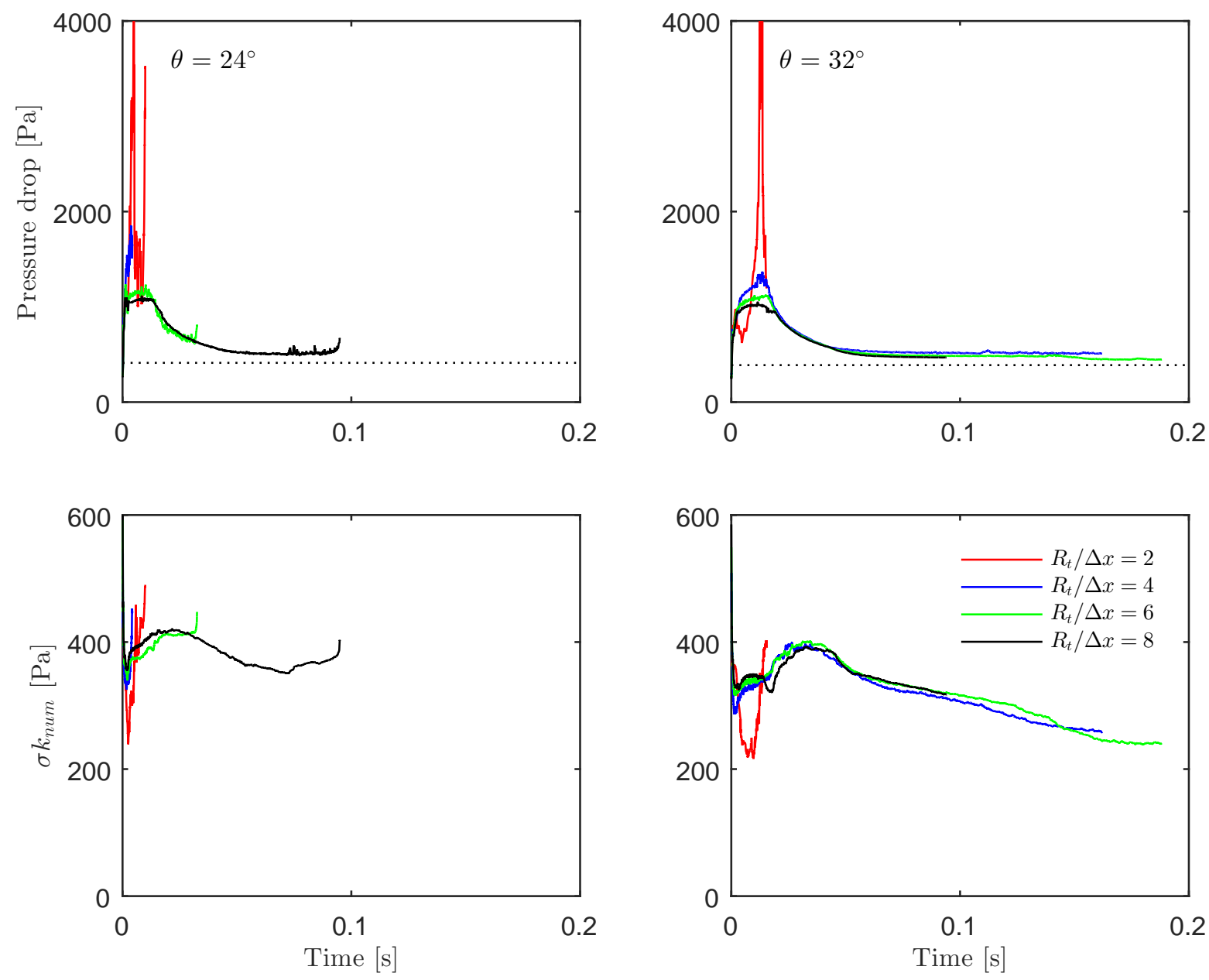

Figure 11. Pressure drop between the inlet and the outlet (top row) and average interface curvature (bottom row) as a function of time for different values of grid resolution and contact angle (case 1.1, square cross-section). Dotted line: entry capillary pressure for an unconstricted pore with infinite length computed using the MS-P method 
occurs or not. The numerical capillary pressure is computed as

$$
p_{c, \text { num }}=\sigma k_{n u m}
$$

where $k_{n u m}$ is computed using eq. (23).

For the case without snap-off, three stages are outlined. Initially, as the bubble of non-wetting phase invades the throat, the pressure rises as a result of the increase in the interface mean curvature. There is a peak in capillary pressure when the bubble assumes its max curvature, corresponding to the point the bubble starts filling the second pore. Then, as the bubble invades the second pore, the interface mean curvature decreases and so does the pressure. Finally the pressure drop approaches the theoretical value of the entry capillary pressure for an unconstricted pore with infinite length computed using the MS-P method (Mayer and Stowe, 1965; Princen, 1969). The case with snap-off is characterized by flow instabilities and thus large variations in capillary pressure. When snap-off occurs, an instantaneous jump in pressure is observed as a result of the bubble break-up. As the figure clearly shows, the predicted pressure drop for the case with coarse resolution can be up to five times as the value predicted by the finer resolution models. These differences tend to diminish as the grid resolution increases, and the solution for the cases with $R_{t} / \Delta x=4,6$ and 8 are very similar for the case without snap-off. These nonphysical pressure values may have an impact when running simulations on digital images of real rock samples. As a result of the segmentation process, the narrower throats, which are the ones determining the permeability of the medium, may potentially be resolved by very few elements, leading to inaccurate estimates of the capillary pressure. A possible remedy is to create finer grids such that the narrower throats are resolved by more than 4 grid cells. This can be done by subdividing each cell into several sub-cells. However, this has two major consequences on the overall computational cost of the simulations. Firstly, increasing the size of the computational domain by a factor of at least 8 increases the computational time required for performing each time step. Secondly, refining the original grid implies using a smaller grid size, leading to a time step smaller by a factor of at least $2^{3 / 2}$, as constrained by eq. (19).

To quantify the difference in accuracy between the different mesh resolutions used, we set the finer resolution model $\left(R_{t} / \Delta x=8\right)$ as the solution, and considered four accuracy 
Table 3. Accuracy measures for different values of contact angle, and for different mesh resolutions (case 1.1, square cross-section).

\begin{tabular}{lllllr}
\hline$\left.\theta^{\circ}\right]$ & $R_{t} / \Delta x$ & $\epsilon_{p}$ & $\bar{\epsilon}_{p}$ & $\epsilon_{k}$ & $\bar{\epsilon}_{k}$ \\
\hline \multirow{2}{*}{24} & 4 & 0.664 & 0.343 & 0.075 & 0.063 \\
& 6 & 0.110 & 0.056 & 0.063 & 0.027 \\
32 & 4 & 0.300 & 0.042 & 0.015 & 0.020 \\
& 6 & 0.070 & 0.017 & 0.020 & 0.015 \\
\hline
\end{tabular}

indicators: the difference in peak pressure $\epsilon_{p}$, the average difference in pressure $\bar{\epsilon}_{p}$, the difference in maximum curvature $\epsilon_{k}$ and the average difference in curvature $\bar{\epsilon}_{k}$. Tab. 3 reports these values for different mesh resolutions. Results show that a resolution of $R_{t} / \Delta x=4$ is enough for accurately represent the interface (all the indicators are always within $10 \%$ of accuracy). However, with this mesh resolution the total pressure drop can be overestimated, especially at snap-off. However, the focus here is on developing understanding of the mechanism, putting the emphasis on capturing the correct physics of the interfacial two-phase flow rather than estimating the pressure peaks. Besides, we are more interested in determining the influence of the variation of the different physical quantities involved, rather than on the exact quantification of the flow variables. Hence, a value of $R_{t} / \Delta x=4$ is sufficient to ensure a good balance between computational effort and results accuracy, and thus is used in the following simulations.

\subsection{Description of the mechanism}

The mechanism is illustrated in Fig. 12 for the case 1.1 of Tab. 2 with square crosssection. Two scenarios, with and without snap-off, are presented, corresponding to values of $\theta$ equal to 30 and $35^{\circ}$ respectively. As the bubble invades the throat (Fig. 12a), the mean curvature of the advancing front starts to increase, and so does the capillary pressure, as a result of the bubble being squeezed into the throat. This curvature (or pressure) drop between the interface advancing front just beyond the throat-pore junction and the initial unconstricted section of the pore causes faster interface propagation within the throat than within the initial uncostricted section. This leads to stretching of the interface, creating a section with much lower radius and hence higher capillary pressure. This in turn initiates some amount of wetting phase to flow back along the corners of the crosssection, as shown in Fig. 13. If this drop in curvature is sufficiently large, enough wetting phase may accumulate in the throat to cause the interfaces from two corner regions to 

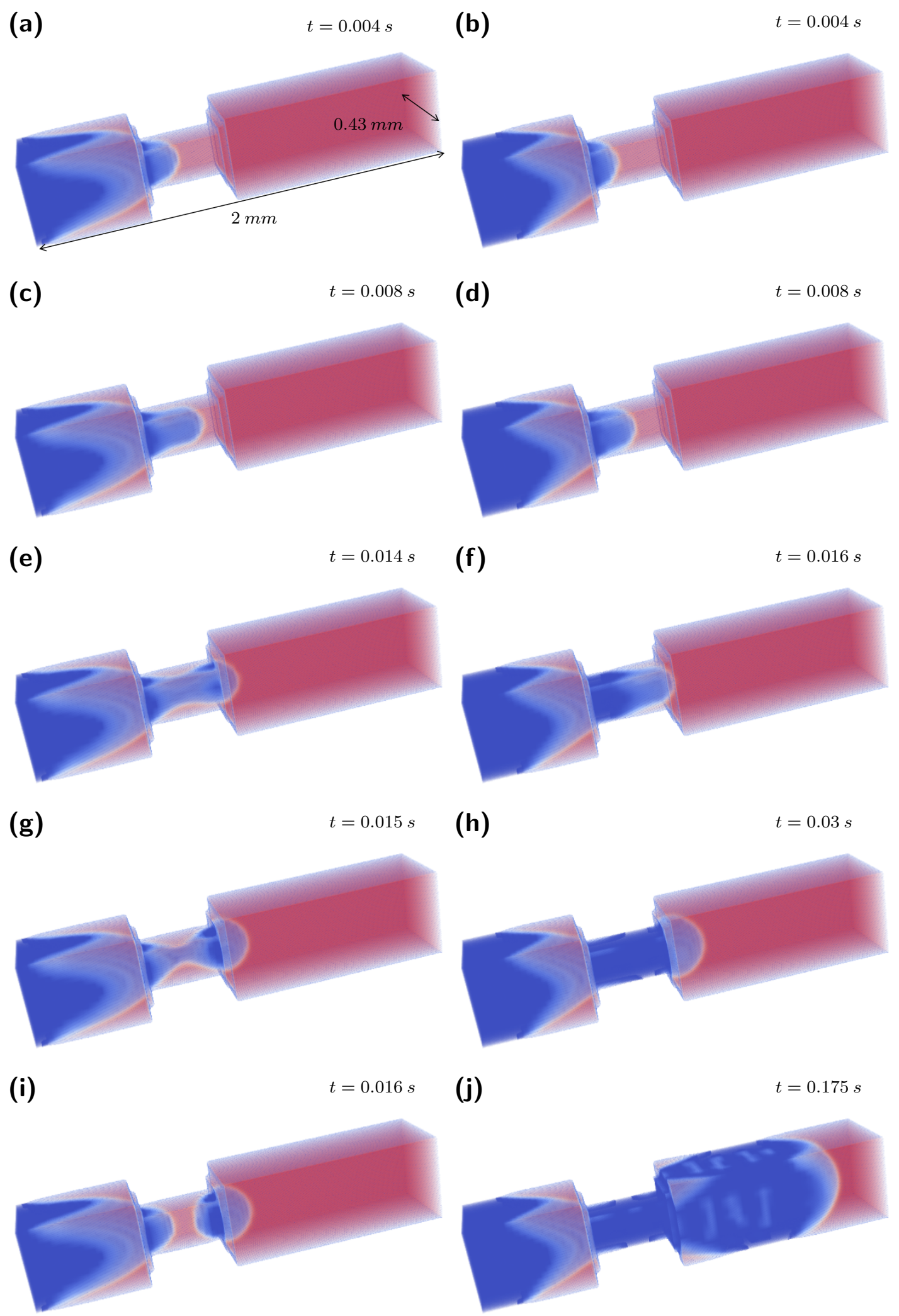

Figure 12. Time evolution of the volume fraction (case 1.1, square cross-section). Left column: $\theta=30^{\circ}$, right column: $\theta=35^{\circ}$. 
(a)

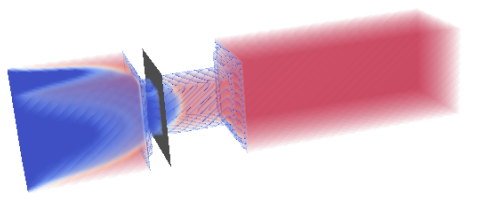

(b)

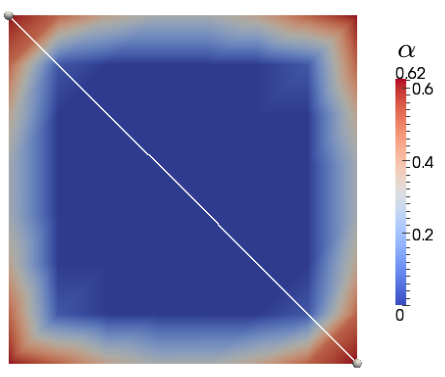

(c)

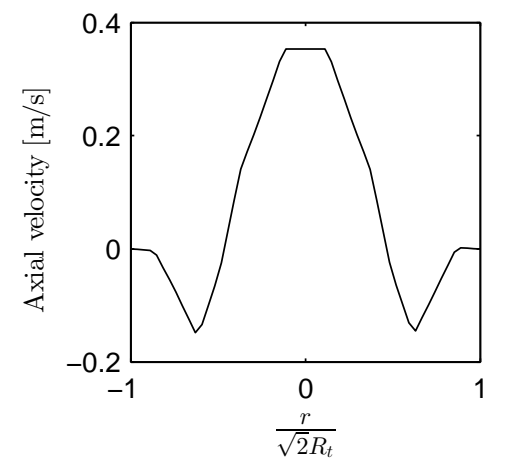

Figure 13. Visualization of the flow-back of the wetting phase along the corners of a square cross-section when the non-wetting phase invades the throat (Fig. 12a). (a) location of the cross-section, (b) volume-fraction distribution in the section and (c) axial velocity along the diagonal line (the x-axis is normalized against the diagonal half-length, where $R_{t}$ is the square half-side and $r$ is the distance from the origin, the latter positioned at the centre of the section).

(a)

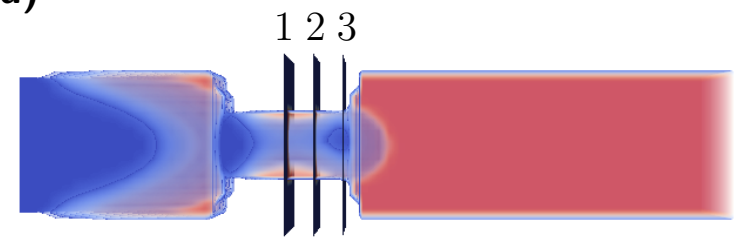

(b)

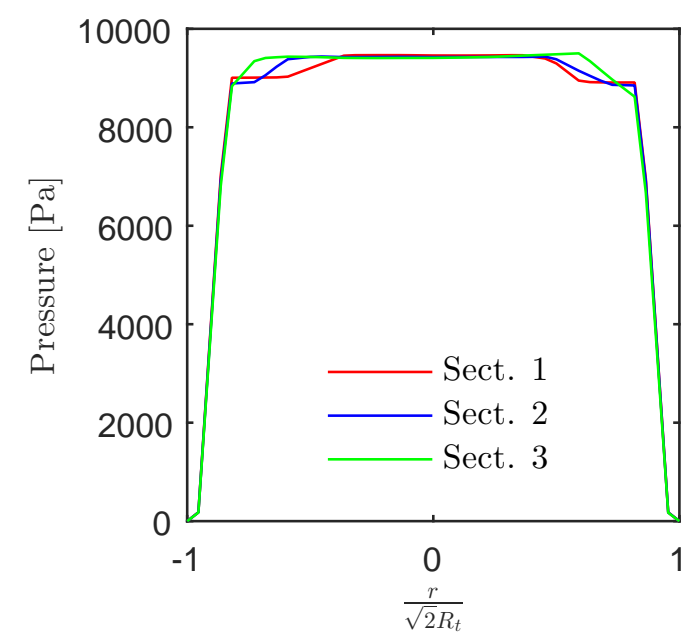

Figure 14. Visualization of the pressure gradient driving the flow-back of the wetting phase along the corners of a square cross-section, when the non-wetting phase invades the second pore (Fig. 12e). (a) location of the cross-sections, (b) pressure along the diagonal line. 


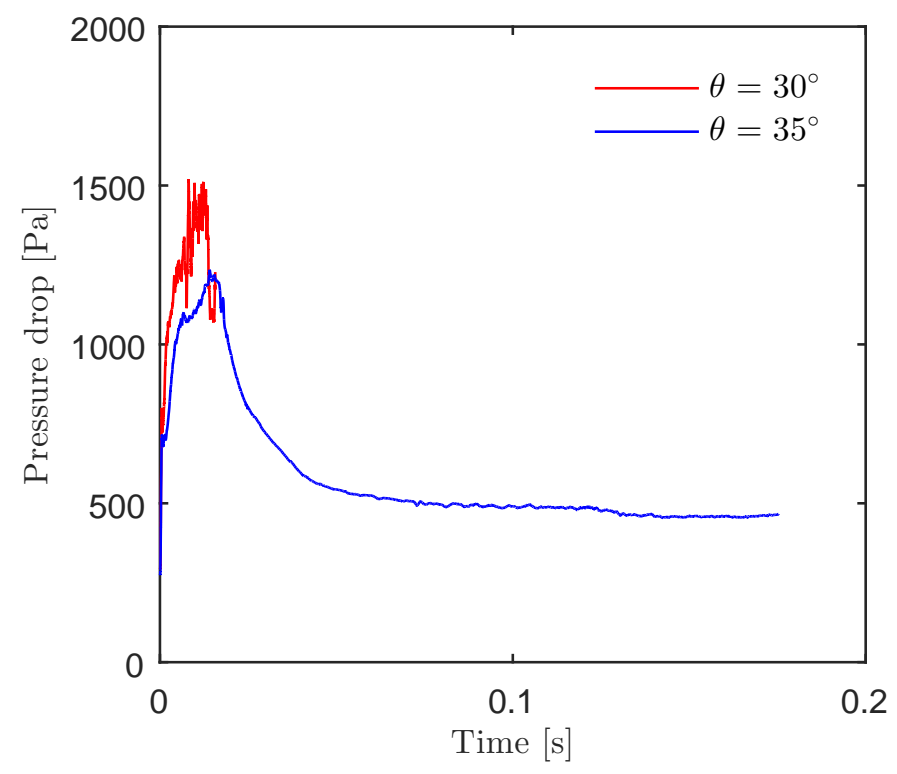

Figure 15. Pressure drop between the inlet and the outlet as a function of time for different values of the contact angle $\theta$ (case 1.1, square cross-section).

meet, and thus the flow to become unstable (Fig. 12c). This flow instability was distinctly observed during the simulations, and it was the critical condition leading to snap-off for long throats $(A R=5)$. Coversely, for shorter throats like the one in Fig. $12(A R=1.5)$, as the bubble reaches the throat exit (Fig: 12e), the interface starts to expand and the curvature decreases. Again, this drop in curvature causes a pressure gradient for the wetting phase. Fig. 14 clearly shows that while the pressure of the injected non-wetting phase remains constant in the central part of the throat, there is a pressure difference at the corners. This pressure difference thus acts as a driving force for the corner flow of the wetting phase from the second pore back into the throat, initiating Rayleigh instability. At this point, snap-off occurs almost instantanously. The wetting phase accumulating in the throat squeezes the collar of injected non-wetting phase (Fig. 12g) until it eventually breaks up, and two separate ganglia of non-wetting phase are formed (Fig. 12i). The newly-formed bubble will be floating within the second pore while the inlet-connected ganglion will re-start the mechanism from a configuration equivalent to (a). The case without snap-off does not present phenomena of flow instability. This is because when the bubble invades the second pore the entire cross-section of the throat is filled with the non-wetting phase, and thus the corner flowback of the wetting phase is prevented (see Fig. 12h). This is because larger contact angles force the interface towards the corners and thus there is less area available for the corner flow back.

These steps are made clearer by plotting the pressure drop between the inlet and the 
outlet as a function of time (see Fig. 15) for both scenarios. The same stages outlined in the previous section apply here. For the case without snap-off, the pressure initially rises as the bubble of non-wetting phase invades the throat (Fig. 12d). The peak in capillary pressure, i.e. the bubble assumes its max curvature, corresponds to the point the bubble starts filling the second pore (see Fig. 12f). Then, as the bubble invades the second pore (Fig. 12h), the interface mean curvature decreases and so does the pressure until it approaches its theoretical value for a single unconstricted pipe.

Conversely, when snap-off occurs, an instantaneous jump in pressure is observed as a result of the bubble break-up. The pressure at the inlet sharply increases, since the inletconnected ganglion is back to the initial phase of invading the throat (see Figs. 12a and 12i), with consequent increase in curvature and capillary pressure. Besides, after snapoff (12i) there are two $n w-w$ interfaces with a large curvature, and therefore a large pressure drop, with in-between a $w-n w$ inteface with the opposite effect but with much smaller curvature. The localization of this pressure jump in the pressure vs time graph can therefore be used to find the exact location where snap-off occurs within the porethroat system. An example of this is shown in Figs. 16 and 17 for case 1.1 with circular cross-section. For $\theta=26^{\circ}$, the pressure drop rises dramatically as the non-wetting fluid invades the throat and the jump occurs at time $t=0.016 \mathrm{~s}$ before the curve approaches its descending branch, that is snap-off takes place at the throat. Conversely, for $\theta=28^{\circ}$, the pressure jump occurs at time $t=0.09 \mathrm{~s}$ when the curve is in its descending branch, that is snap-off occurs after the non-wetting bubble has already invaded the second pore. Finally, for $\theta=30^{\circ}$, snap-off does not occur.

\subsection{Effects of contact angle}

The time required for snap-off as a function of the contact angle for all the geometries considered is shown in Fig. 18. These graphs reveal two major findings. Firstly, the time required for snap-off increases with the contact angle. This can be easily explained by the fact that larger contact angles increase the flow resistence by forcing the interface towards the walls and thus reducing the available area for the flow back of the wetting phase (Legait, 1983; Ransohoff and Radke, 1988). Obviously, the time required for snapoff is generally much less for the simulations with $C R=3$ that those with $C R=2$. Less 


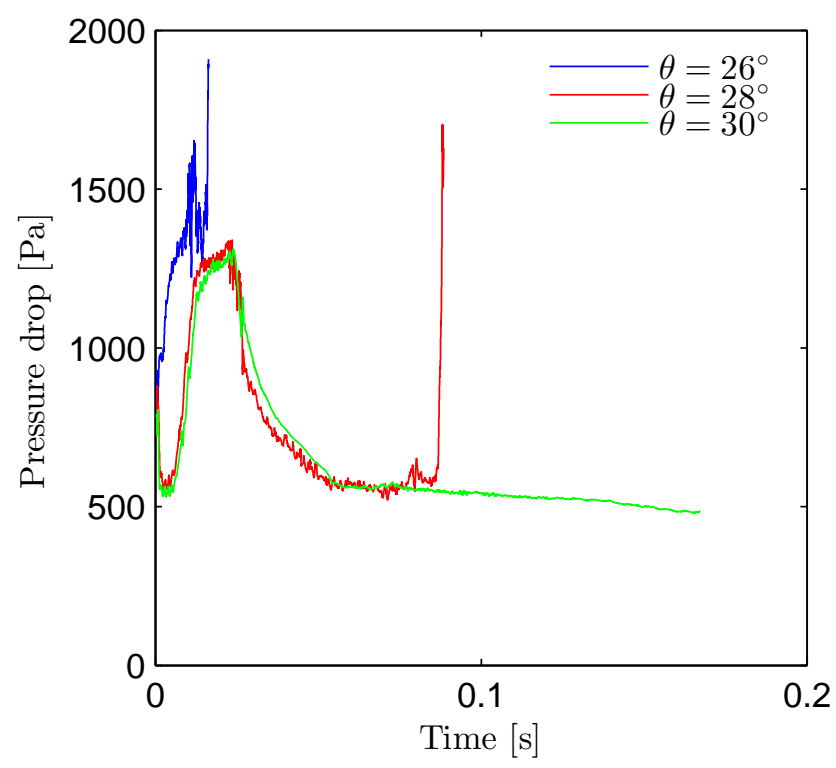

Figure 16. Pressure drop between the inlet and the outlet as a function of time for different values of the contact angle $\theta$ (case 1.1, circular cross-section).

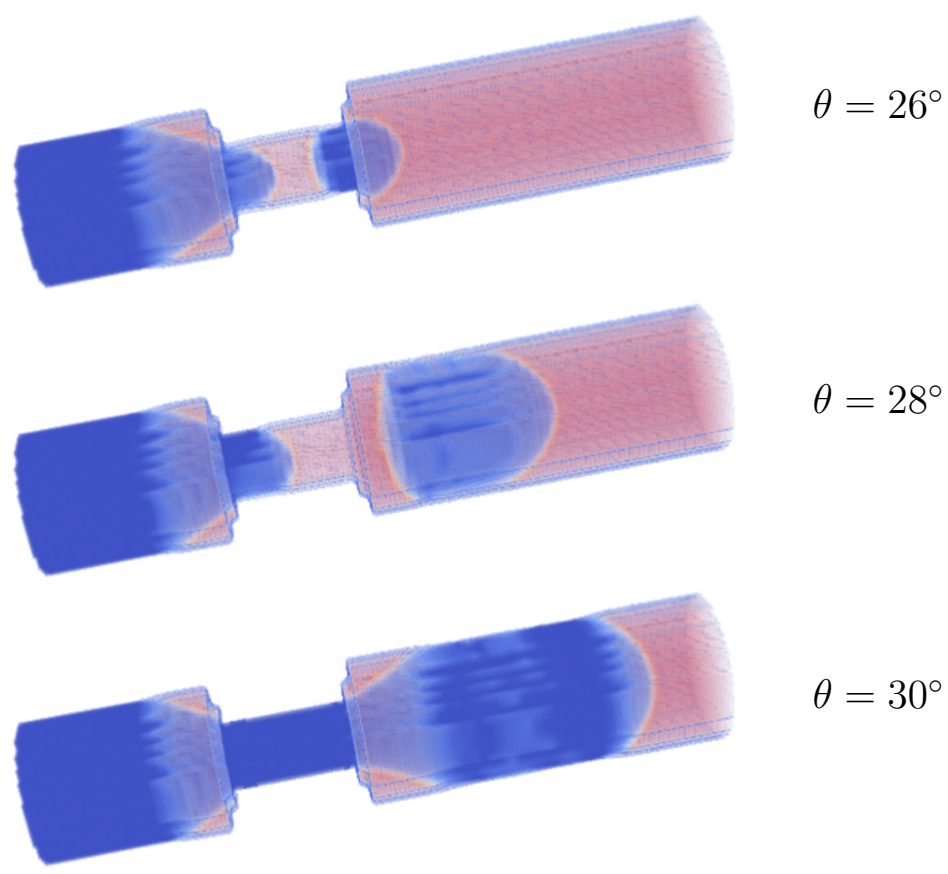

Figure 17. Volume fraction distribution at the time of instantaneous snap-off, when occurring, for different values of the contact angle $\theta$ (case 1.1, circular cross-section). 

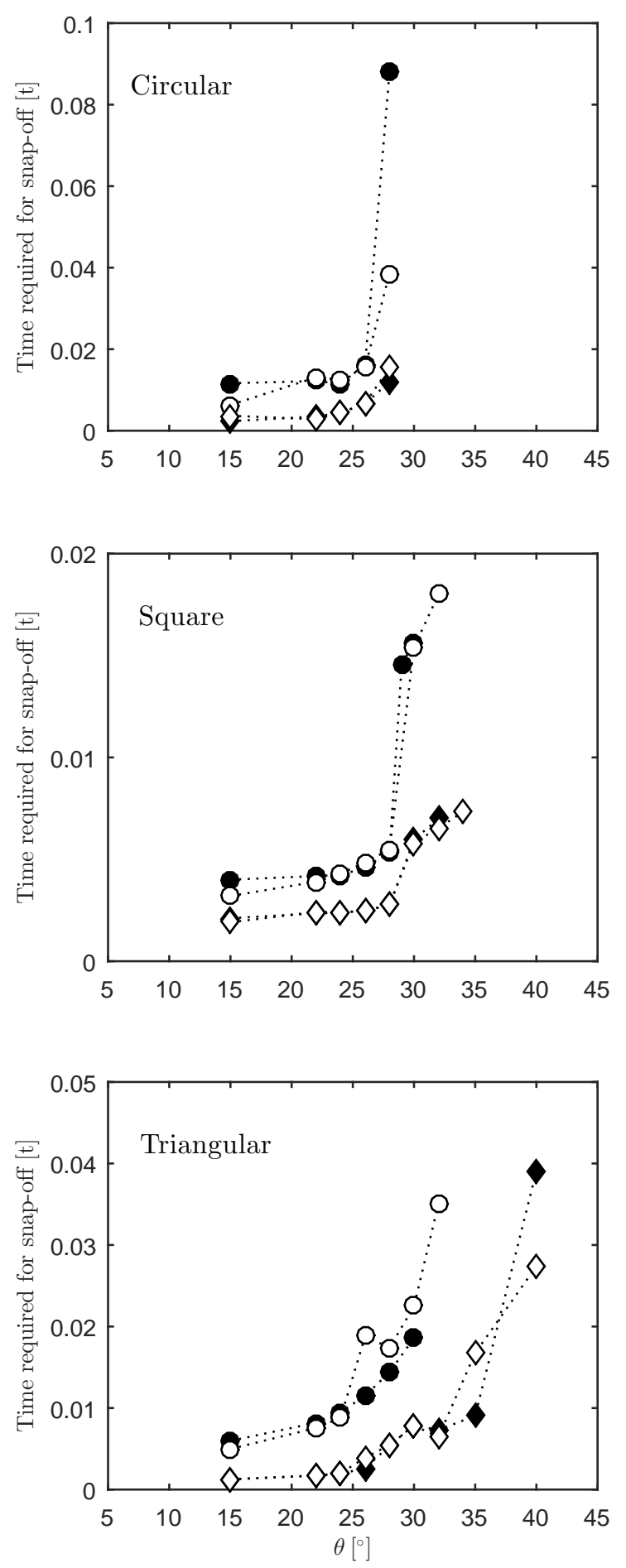

Figure 18. Time required for snap-off as a function of the contact angle for all the geometries considered. Legend: case 1.1 - black-filled circle; case 1.2 - black-filled diamond; case 1.3 - white-filled circle; case 1.4 - white-filled diamond. 
obviously, no significant changes in the time required for snap-off are observed for the case with longer throats.

The second aspect to highlight is that $\theta_{\text {thresh }}$ increases with diminishing the roundness of the cross-section, passing from a value of $28^{\circ}$ for the circular cross-section to values of $30-34^{\circ}$ and up to $40^{\circ}$ for the square and triangular cross-section respectively. This can be explained by using the concept of dimensionless flow resistence (Ransohoff and Radke, 1988). The dimensionless flow resistence is a parameter describing the wetting flow along the corners of a non-circular conduit and is determined solely by the geometry of the corner (half-angle and roundness). For a given interface radius of curvature, as the corner half-angle increases, i.e. from $30^{\circ}$ of the equilateral triangular cross-section to $45^{\circ}$ of the rectangular one, the area available for the wetting flow along the corner decreases, resulting in a greater flow resistance. This greater flow resistence therefore acts as an impediment to the flow of the wetting phase, reducing the area available for the flow back along the corners of the cross-section, which is the origin of the snap-off mechanism. The circular cross-section can be seen as a limiting condition with an halfangle equal to $90^{\circ}$. However, with the approximations introduced when discretizing a circle using a structured cubic mesh, the circular cross section becomes in practice an irregular polygon. Nevertheless, since the aim here is to simulate pore-scale events taking place in real porous microstructures, the case with perfectly smoothed rounded capillary tubes of circular cross-section has no practical relevance. However, circular cross-sections are used in pore-network modelling for fairly smooth pores with high shape factors, whereas more irregular pore shapes are usually represented by square and triangular cross-sections with very sharp corners, such as the ones used in this study.

\subsubsection{Entry capillary pressure}

In this section, we compare the throat entry capillary pressure computed during the simulations with the theoretical values for a pore with the same cross-section and infinite length computed using the MS-P method (Mayer and Stowe, 1965; Princen, 1969). For a circular cross-section the theoretical value of the entry capillary pressure is computed from the curvature given by eq. (25). For the two angular cross-sections, the entry capillary 

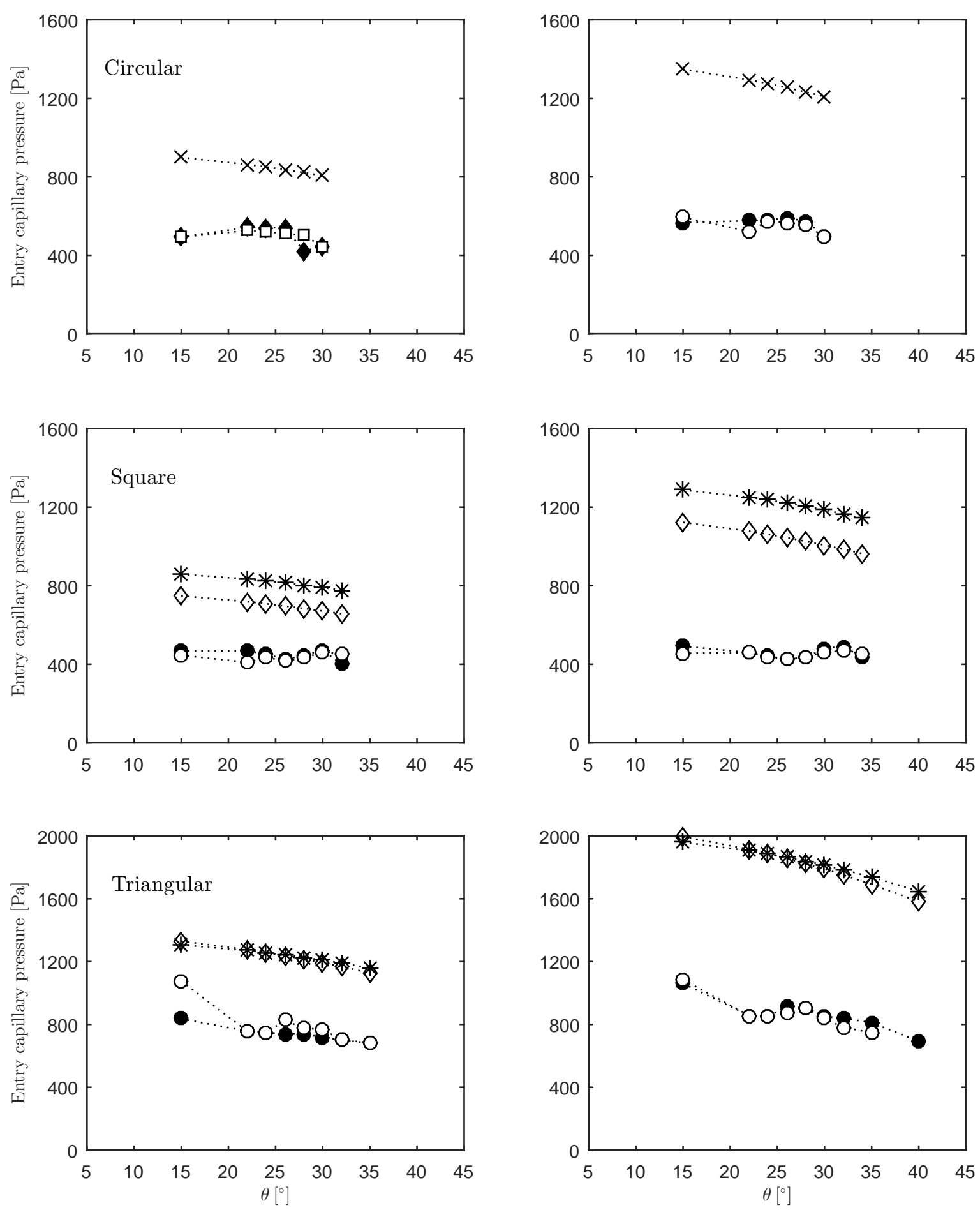

Figure 19. Entry capillary pressure as a function of contact angle for all the geometries considered. Legend: cross - theoretical value for circular pores, eq. (25); asterisk - MS-P method, eq. (31); diamond - model by Rabbani et al. (2016), eq. (33); black-filled circle - numerical value for $\mathrm{AR}=1.5$; white-filled circle - numerical value for $\mathrm{AR}=5$. 
pressure is computed using the following expression:

$$
p_{c}=\frac{\sigma(1+2 \sqrt{\pi G} \cos \theta)}{r_{i}} F,
$$

where $r_{i}$ is the inscribed radius of the pore, $\mathrm{G}$ is the shape factor defined as the ratio between the cross-sectional area and the square of the perimeter, and $\mathrm{F}$ is a factor computed as follows

$$
F=\frac{1+\sqrt{1+\frac{4 G H}{\cos ^{2} \theta}}}{1+2 \sqrt{\pi G}},
$$

where

$$
H=\pi\left(1-\frac{\theta}{\pi / 3}\right)+3 \sin \theta \cos \theta-\frac{\cos ^{2} \theta}{4 G}
$$

We also compare the entry capillary pressure against an empirical formulation given by Rabbani et al. (2016) for angular pore shapes:

$$
p_{c}=\frac{2 \pi r_{i} \sigma \cos \theta\left(1-\frac{\beta}{\pi}\right)}{G P^{2}} \frac{\sin (\pi-2 \beta)}{\sin \beta},
$$

where $P$ is the cross-section perimeter and $\beta$ is the half corner angle.

The comparison between the different entry capillary pressure measures for all the geometries considered is presented in Fig. 19. The numerical value is the maximum value of $p_{c, \text { num }}$, computed using eq. (30), recorded during the simulation. The discrepancy between theoretical and numerical values is due to two main reasons. Firsly, theoretical values are computed for equilibrium conditions. We have already shown in Sec. 3.2 that under these conditions the interface curvature is predicted accurately by the model. During snap-off instead, the interface is accelerated forward and local capillary numbers are very high. Inertial effects, neglected by theoretical models, are here accounted for in the simulations. Secondly, while theoretical values are computed for pores of constant cross-section and infinite length, here we have channels of variable cross-section, thus the interface has different curvature along its whole longitudinal profile. Averaging over the whole interface region implies that the interface curvature is determined not exclusively by the larger curvature of the interface front within the throat, but also the interfaces with smaller curvature in the pore-throat junction are accounted for, resulting in a smaller 


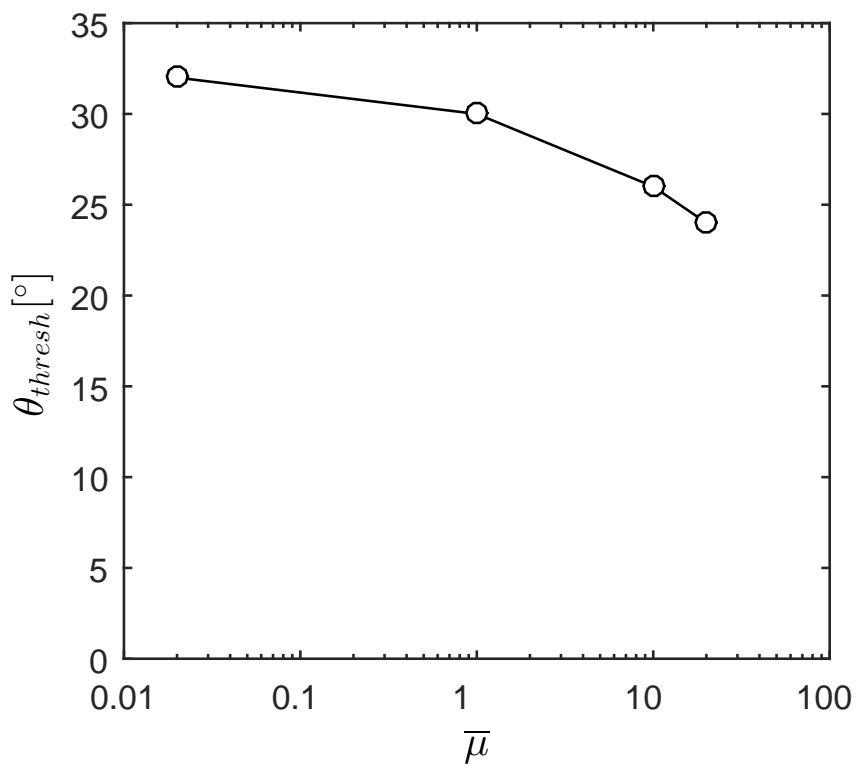

Figure 20. Predicted values of the threshold contact angle as a function of the viscosity ratio $\bar{\mu}=\frac{\mu_{n w}}{\mu_{w}}$ (case 2$)$.

average capillary pressure. The same discrepancy was found in the work of Rabbani et al. (2016).

\subsection{Effects of viscosity ratio}

The predicted values of the threshold contact angle $\theta_{\text {thresh }}$ as a function of the viscosity ratio are shown in Fig. 20. As the graph clearly shows, $\theta_{\text {thresh }}$ decreases with increasing the viscosity ratio. This is due to the increase in the viscous forces of the non-wetting injected fluid relative to capillary forces when $\bar{\mu}$ increases. These forces oppose the flow of the injected more viscous phase, hence they also act as an impedement to the corner flow back of the resident wetting phase. As a result, when the non-wetting phase invades the throat, the interface assumes a stable configuration by filling the entire cross-section. Hence, smaller values of the contact angle, i.e. more bending of the interface, are necessary for increasing the area available for the wetting corner flow and therefore inducing the flow instability that leads to snap-off. Fig. 21 clearly shows this mechanism. When $\bar{\mu}=1$, velocity is negative near the corners, meaning that the corner flow back of the wetting phase is initiated. Conversely, for a higher viscosity ratio, velocity is always positive. However, there is an increase of velocity at the corners, which are still occupied by the resident wetting phase. This is probably due to the fact that capillary forces are responsible for deviating the path of the fluid particles, aligning them with the interface. 
As a result the axial velocity in the transition region where the interface resides is very low. In pratice, the velocity profile for the case with $\bar{\mu}=1$ is a dumped version of the one with $\bar{\mu}=10$, with viscous forces preventing the sharpness of the velocity gradients. Reduction in intensity and in some cases even suppression of snap-off due to high oil viscosity was also observed experimentally (Peña et al., 2009). This is of paramount importance for practical applications such as enhanced oil recovery and all industrial processing involving emulsions. In particular it provides explanation for high efficiency oil recovery when polymer-coated nanoparticles (PNPs) are used (ShamsiJazeyi et al., 2014).

Fig. 22 shows the time required for snap-off as a function of the viscosity ratio for a fixed value of $\theta=24^{\circ}$, corresponding to the threshold value of the contact angle for which snap-off takes place for the whole range of viscosity ratios considered. This increase in time required for snap off for increasing viscosity ratios can be interpreted as an increase of the flow resistence due to the increase of the viscous friction, in agreement with the work of Legait (1983) for square cross-section. Fig. 23 shows the pressure drop as a function of the viscosity ratio for the same set of simulations. This graph illustrates the two main differences between the cases with $\bar{\mu}<1$ and $\bar{\mu}>1$. Firstly, the peak in pressure is significantly greater for higher viscosity ratio. However, this may also be due to numerical instabilities. Secondly, when $\bar{\mu}<1$, the invading fluid fills the throat very rapidly as there is less flow resistance and thus no impedement to the corner flow back of the wetting phase. On the contrary, when the invading fluid is more viscous than the resident fluid, the time required to invade the throat is much higher, again due to the increase in the viscous resistance. This can be distinctly observed in fig. 23 from the duration of the stage where the pressure drop is roughly constant, i.e. before the pressure drop starts decreasing when the injected fluid invades the second pore. This plateau becomes progresssively longer with increasing the viscosity ratio. However, snapoff occurs after the fluid has invaded the second pore for all cases.

Finally, we note that when the injected fluid is up to two-order of magnitude less viscous than the resident fluid, the time required for snap-off does not significantly differ from the case with $\bar{\mu}=1$. This may be of help for simulations at real reservoir conditions for practical engineering applications, such as injection of $\mathrm{CO}_{2}$ in water/brine saturated rocks. Results shown in Fig. 22 indicate that for this pair of fluids, one may reasonably 


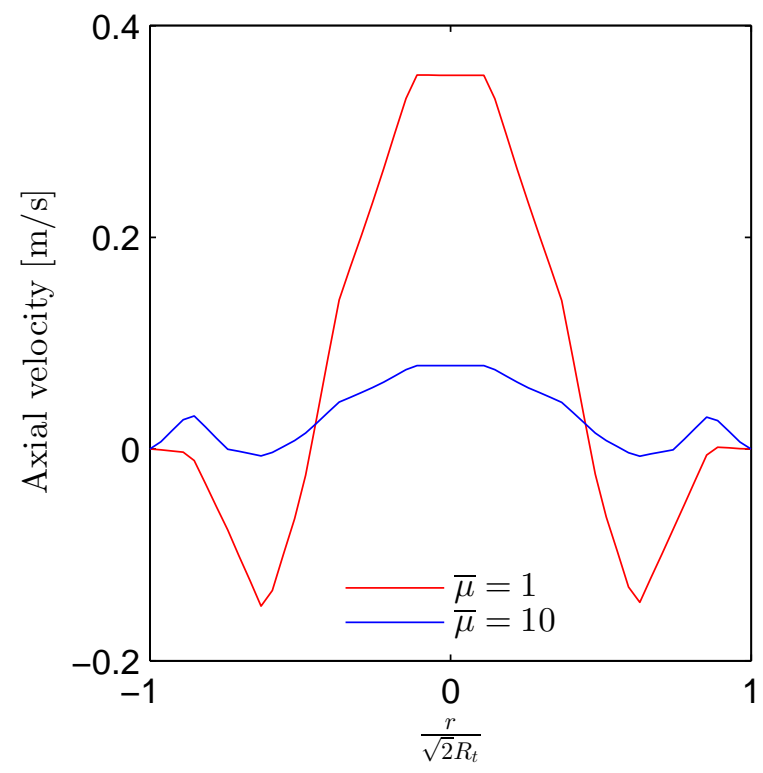

Figure 21. Axial velocity along the diagonal of a cross-section taken at the same position and at the same time step as in Fig. 13a, for different viscosity ratios (case $2, \theta=30^{\circ}$ ).

take the viscosity ratio equal to one as first approximation, and thus reduce the numerical instabilities, i.e. vortices, which are likely to be induced by high viscosity ratios (see Sec. $3.2)$.

\section{Summary and conclusions}

A stable and accurate FV-based algorithm for modelling two-phase flow in porous media at the pore-scale has been developed.

We applied the algorithm to study the snap-off mechanism. We simulated drainage in a single pore-throat constriction of variable geometry and for different shapes of the cross-sections, and investigated the role of several parameters such as contact angle, viscosity ratio and capillary number. Numerical simulations were shown to be a very useful means for understanding the dynamics of this mechanism, providing information on the instantaneous distribution of the relevant quantities otherwise impossible to access using traditional experiments. Snapshots of the velocity field clearly showed that snap-off is caused by local instability of the front meniscus, when the pore-throat constriction is such that a corner flow-back of the wetting phase is initiated.

Preliminary simulations revealed that poor resolution of the narrower throat can lead to a non-physical building-up of the pressure field. These non-physical pressure values may have an impact when running simulations on digital images of real rock samples. As 


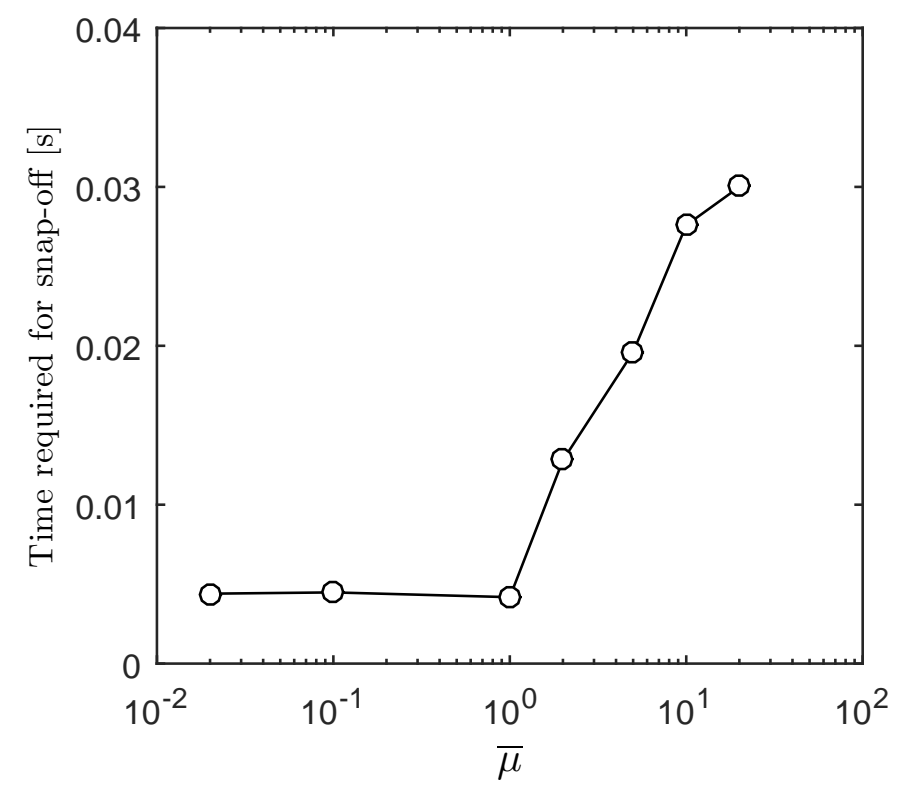

Figure 22. Time required for snap-off as a function of the viscosity ratio (case $2, \theta=24^{\circ}$ ).

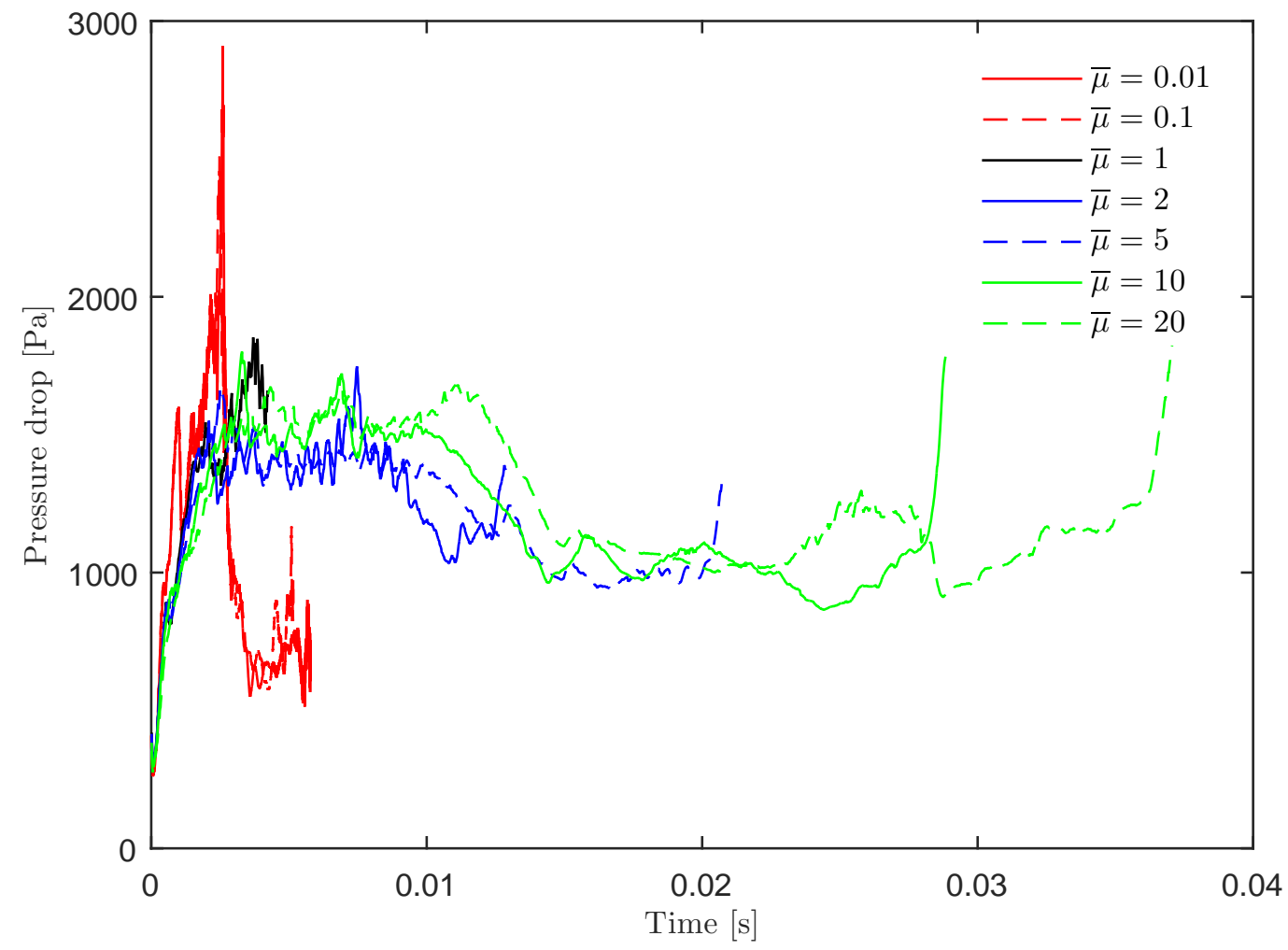

Figure 23. Pressure drop between the inlet and the outlet as a function of time for different values of viscosity ratio $\bar{\mu}$ (case $2, \theta=24^{\circ}$ ). 
a result of the segmentation process, the narrower throats, which are the ones determining the permeability of the medium, may potentially be resolved by very few elements, leading to inaccurate estimates of the capillary pressure.

The study highlighted the role of contact angle and viscosity ratio in determining the dynamics of snap-off. The threshold contact angle for snap-off, $\theta_{\text {thresh }}$, i.e. the value of the static contact angle above which snap-off is inhibited, has been computed for each case. Results showed that $\theta_{\text {thresh }}$ increases with dimininshing the roundness of the crosssection, passing from a value of $28^{\circ}$ for the circular cross-section to values of $30-34^{\circ}$ for the square cross-section, and up to $40^{\circ}$ for the triangular cross-section. The analysis of the viscosity ratio has been restricted to the single case with square cross-section, $A R=1.5$ and $C R=2$. Increasing the viscosity of the injected phase resulted in a drop in $\theta_{\text {thresh }}$ from a value of $30^{\circ}$ when $\bar{\mu}=1$ to a value of $26^{\circ}$ when $\bar{\mu}=10$ and down to a value of $24^{\circ}$ when $\bar{\mu}=20$. On the contrary, when the viscosity of the injected fluid is up to two-order of magnitude less than that of the resident fluid, there is no significant difference with the case with $\bar{\mu}=1$.

\section{References}

Aris, R. (1962). Vectors, tensors and the basic equations of fluid mechanics. Prentice Halls, Englewood Cliffs.

Armstrong, R., Berg, S., Dinariev, O., Evseev, N., Klemin, D., Koroteev, D., and Safonov, S. (2016). Modeling of pore-scale two-phase phenomena using density functional hydrodynamics. Transport in Porous Media, 112(3):577-607.

Beresnev, I. A. and Deng, W. (2010). Theory of breakup of core fluids surrounded by a wetting annulus in sinusoidally constricted capillary channels. Physics of Fluids, 22(1):012105.

Beresnev, I. A., Li, W., and Vigil, R. D. (2009). Condition for break-up of non-wetting fluids in sinusoidally constricted capillary channels. Transport in porous media, 80(3):581.

Blunt, M. J. et al. (1997). Effects of heterogeneity and wetting on relative permeability using pore level modeling. SPE Journal, 2(01):70-87.

Brackbill, J., Kothe, D. B., and Zemach, C. (1992). A continuum method for modeling surface tension. Journal of computational physics, 100(2):335-354. 
Brackbill, J. U. and Kothe, D. B. (1996). Dynamical modeling of surface tension. In NASA Conference Publication, pages 693-700.

Deng, W., Cardenas, M. B., and Bennett, P. C. (2014). Extended roof snap-off for a continuous nonwetting fluid and an example case for supercritical co 2. Advances in Water Resources, 64:34-46.

Dimitrov, D., Milchev, A., and Binder, K. (2007). Capillary rise in nanopores: molecular dynamics evidence for the lucas-washburn equation. Physical review letters, 99(5):054501.

Gauglitz, P. and Radke, C. (1990). The dynamics of liquid film breakup in constricted cylindrical capillaries. Journal of Colloid and Interface Science, 134(1):14-40.

Gueyffier, D., Li, J., Nadim, A., Scardovelli, R., and Zaleski, S. (1999). Volume-of-fluid interface tracking with smoothed surface stress methods for three-dimensional flows. Journal of Computational physics, 152(2):423-456.

Hirt, C. W. and Nichols, B. D. (1981). Volume of fluid (vof) method for the dynamics of free boundaries. Journal of computational physics, 39(1):201-225.

Huppert, H. E. and Neufeld, J. A. (2014). The fluid mechanics of carbon dioxide sequestration. Annual Review of Fluid Mechanics, 46:255-272.

Issa, R. I. (1986). Solution of the implicitly discretised fluid flow equations by operatorsplitting. Journal of computational physics, 62(1):40-65.

Kothe, D., Rider, W., Mosso, S., Brock, J., and Hochstein, J. (1996). Volume tracking of interfaces having surface tension in two and three dimensions. AIAA paper, 96(0859):118.

Kovscek, A. and Radke, C. (1996). Gas bubble snap-off under pressure-driven flow in constricted noncircular capillaries. Colloids and Surfaces A: Physicochemical and Engineering Aspects, 117(1):55-76.

Lafaurie, B., Nardone, C., Scardovelli, R., Zaleski, S., and Zanetti, G. (1994). Modelling merging and fragmentation in multiphase flows with surfer. Journal of Computational Physics, 113(1):134-147.

Legait, B. (1983). Laminar flow of two phases through a capillary tube with variable square cross-section. Journal of colloid and interface science, 96(1):28-38.

Levine, S., Lowndes, J., Watson, E. J., and Neale, G. (1980). A theory of capillary rise of a liquid in a vertical cylindrical tube and in a parallel-plate channel: Washburn equation 
modified to account for the meniscus with slippage at the contact line. Journal of Colloid and Interface Science, 73(1):136-151.

Mayer, R. P. and Stowe, R. A. (1965). Mercury porosimetrybreakthrough pressure for penetration between packed spheres. Journal of colloid Science, 20(8):893-911.

Mogensen, K. and Stenby, E. H. (1998). A dynamic two-phase pore-scale model of imbibition. Transport in Porous Media, 32(3):299-327.

Peña, T., Carvalho, M., and Alvarado, V. (2009). Snap-off of a liquid drop immersed in another liquid flowing through a constricted capillary. AIChE journal, 55(8):1993-1999.

Press, W. H., Teukolsky, S. A., Vetterling, W. T., and Flannery, B. P. (1996). Numerical recipes in $C$, volume 2 . Citeseer.

Princen, H. (1969). Capillary phenomena in assemblies of parallel cylinders: I. capillary rise between two cylinders. Journal of Colloid and Interface Science, 30(1):69-75.

Qaseminejad Raeini, A. (2013). Modelling multiphase flow through micro-CT images of the pore space. $\mathrm{PhD}$ thesis, Imperial College London.

Rabbani, H. S., Joekar-Niasar, V., and Shokri, N. (2016). Effects of intermediate wettability on entry capillary pressure in angular pores. Journal of colloid and interface science, $473: 34-43$.

Raeini, A. Q., Bijeljic, B., and Blunt, M. J. (2014). Numerical modelling of sub-pore scale events in two-phase flow through porous media. Transport in porous media, 101(2):191213.

Raeini, A. Q., Blunt, M. J., and Bijeljic, B. (2012). Modelling two-phase flow in porous media at the pore scale using the volume-of-fluid method. Journal of Computational Physics, 231(17):5653-5668.

Ransohoff, T., Gauglitz, P., and Radke, C. (1987). Snap-off of gas bubbles in smoothly constricted noncircular capillaries. AIChE Journal, 33(5):753-765.

Ransohoff, T. and Radke, C. (1988). Laminar flow of a wetting liquid along the corners of a predominantly gas-occupied noncircular pore. Journal of Colloid and Interface Science, 121(2):392-401.

Renardy, Y. and Renardy, M. (2002). Prost: a parabolic reconstruction of surface tension for the volume-of-fluid method. Journal of Computational Physics, 183(2):400-421.

Rider, W. J. and Kothe, D. B. (1998). Reconstructing volume tracking. Journal of computational physics, 141(2):112-152. 
Roof, J. et al. (1970). Snap-off of oil droplets in water-wet pores. Society of Petroleum Engineers Journal, 10(01):85-90.

Rossen, W. R. (2000). Snap-off in constricted tubes and porous media. Colloids and Surfaces A: Physicochemical and Engineering Aspects, 166(1):101-107.

Rossen, W. R. (2003). A critical review of roof snap-off as a mechanism of steady-state foam generation in homogeneous porous media. Colloids and Surfaces A: Physicochemical and Engineering Aspects, 225(1):1-24.

Saad, Y. (2003). Iterative methods for sparse linear systems. Siam.

Scardovelli, R. and Zaleski, S. (1999). Direct numerical simulation of free-surface and interfacial flow. Annual review of fluid mechanics, 31(1):567-603.

ShamsiJazeyi, H., Miller, C. A., Wong, M. S., Tour, J. M., and Verduzco, R. (2014). Polymer-coated nanoparticles for enhanced oil recovery. Journal of Applied Polymer Science, 131(15).

Sussman, M. and Ohta, M. (2006). High-order techniques for calculating surface tension forces. In Free Boundary Problems, pages 425-434. Springer.

Williams, M., Kothe, D., and Puckett, E. (1998). Accuracy and convergence of continuum surface tension models. Fluid Dynamics at Interfaces, Cambridge University Press, Cambridge, pages 294-305.

Youngs, D. L. (1982). Time-dependent multi-material flow with large fluid distortion. Numerical methods for fluid dynamics, 24(2):273-285.

Yu, L. and Wardlaw, N. C. (1986). The influence of wettability and critical pore-throat size ratio on snapoff. Journal of Colloid and Interface Science, 109(2):461-472. 